\title{
RESTRICTIVE METRIC REGULARITY AND GENERALIZED DIFFERENTIAL CALCULUS IN BANACH SPACES
}

\author{
BORIS S. MORDUKHOVICH and BINGWU WANG
}

Received 20 May 2004

\begin{abstract}
We consider nonlinear mappings $f: X \rightarrow Y$ between Banach spaces and study the notion of restrictive metric regularity of $f$ around some point $\bar{x}$, that is, metric regularity of $f$ from $X$ into the metric space $E=f(X)$. Some sufficient as well as necessary and sufficient conditions for restrictive metric regularity are obtained, which particularly include an extension of the classical Lyusternik-Graves theorem in the case when $f$ is strictly differentiable at $\bar{x}$ but its strict derivative $\nabla f(\bar{x})$ is not surjective. We develop applications of the results obtained and some other techniques in variational analysis to generalized differential calculus involving normal cones to nonsmooth and nonconvex sets, coderivatives of set-valued mappings, as well as first-order and second-order subdifferentials of extended real-valued functions.
\end{abstract}

2000 Mathematics Subject Classification: 49J52, 49J53, 58C20, 90C31.

1. Introduction and preliminaries. This paper is devoted to metric regularity and the generalized differentiation theory in variational analysis which has been well recognized as a fruitful area in mathematics mostly oriented in optimization-related problems and their applications. On the other hand, variational principles and methods of variational analysis are widely applied to the study of a broad range of problems that may not be of a variational nature. We refer the reader to the book by Rockafellar and Wets [33] for a systematic exposition of the key features of variational analysis in finite dimensions.

Since nonsmooth objects (sets with nonsmooth boundaries, set-valued mappings, and extended real-valued functions) appear naturally and frequently in constrained optimization and related areas, generalized differentiation is one of the major parts of variational analysis. In this paper, we focus on Fréchet-like generalized differential constructions and their sequential limits that play an important role in nonsmooth variational analysis and its applications; see, respectively, [4, 18, 20, 25, 33] with the references and commentaries therein for developments and applications in finitedimensional and infinite-dimensional spaces.

Most of the results previously obtained for the mentioned constructions require that the Banach spaces in question be Asplund, that is, every separable subspace of them have a separable dual. This includes all spaces with Fréchet differentiable renorms or bump functions, in particular, every reflexive Banach space. On the other hand, there are important Banach spaces that are not Asplund (e.g., the classical functional spaces $L^{1}, L^{\infty}$, and $C$ ). In what follows, we are not going to impose the Asplund property and we will consider the general Banach space setting. 
The results obtained in this paper concern first-order and second-order calculus; the latter deals with chain rules for second-order subdifferentials. An important property of mappings used in the development of generalized differential calculus is the so-called metric regularity. Recall that a mapping $f: X \rightarrow Y$ between Banach spaces is metrically regular around $\bar{x}$ if there are $\mu>0$ and neighborhoods $U$ of $\bar{x}$ and $V$ of $\bar{y}:=f(\bar{x})$ such that

$$
\operatorname{dist}\left(x ; f^{-1}(y)\right) \leq \mu\|f(x)-y\| \quad \forall x \in U, y \in V \text {. }
$$

The celebrated Lyusternik-Graves theorem ensures this property for a mapping $f$ strictly differentiable at $\bar{x}$ with the surjective derivative $\nabla f(\bar{x}): X \rightarrow Y$. Moreover, the surjectivity of $\nabla f(\bar{x})$ is also necessary for the metric regularity of $f$ around $\bar{x}$. We refer the reader to the original papers of Lyusternik [17] and Graves [9], as well as to the recent discussions in $[7,11]$ and the works therein.

It is valuable for the theory and applications to relax the surjectivity assumption and to extend a class of mappings for which one can use metric regularity techniques. Since the surjectivity of $\nabla f(\bar{x})$ is necessary for the metric regularity of strict differentiable mappings, one has to modify the above concept to cover mappings with nonsurjective derivatives. In what follows, we consider the metric regularity for mappings $f$ from $X$ into the image space $f(X)$, which is a metric space, but in general is far from being Banach, and call this notion the restrictive metric regularity (RMR) of $f$ around $\bar{x}$.

Section 2 is devoted to the study of RMR for mappings $f: X \rightarrow Y$ between Banach spaces $X$ and $Y$. Although the RMR property concerns in fact the metric regularity of $f: X \rightarrow E$ with the metric space $E:=f(X) \subset Y$ and hence can be treated by the metric space regularity theory (cf. $[6,11]$ ), we take an advantage of using the Banach space structure on $X$ and $Y$ as well as the strict differentiability of mappings when it applies. In this way, we establish relationships between RMR of nonlinear (generally nonsmooth) mappings and their linear approximations, derive necessary and sufficient conditions for the RMR property via approximations, and obtain efficient criteria for RMR of strictly differentiable mappings with nonsurjective derivatives that extend the Lyusternik-Graves theorem to such mappings important for applications.

In Section 3, we give applications of the RMR property and related results to firstorder calculus rules for generalized normals, coderivatives, and subgradients of sets, set-valued mappings, and extended real-valued functions in Banach spaces. Most of the calculus rules obtained are new even under surjectivity assumptions, ensuring the classical metric regularity of mappings involved in compositions. The principal firstorder results concern computing generalized normals to inverse images of sets under strictly differentiable mappings with possibly nonsurjective derivatives; related chain rules for coderivatives and subgradients follow from them via a geometric approach.

Section 4 concerns chain rules for two kinds of second-order subdifferentials ("normal" and "mixed") generated by the corresponding coderivatives of first-order subgradient mappings. We derive an exact formula for computing mixed second-order subdifferentials and obtain an efficient upper estimate for normal ones, which becomes an equality under natural assumptions discussed below (in particular, when the domain space $X$ is reflexive). 
Finally, Section 5 contains applications of RMR and calculus rules developed in Section 3 to the so-called sequential normal compactness (SNC) properties of sets, setvalued mappings, and extended real-valued functions in infinite dimensions. The latter properties are automatic in finite-dimensional spaces while playing a crucial role in infinite-dimensional variational analysis; see more discussions below. We obtain efficient conditions ensuring the preservation of the SNC properties under some compositions involving RMR mappings.

Our notation is basically standard; compare [25, 33]. Unless otherwise stated, all the spaces considered are Banach, with the norm $\|\cdot\|$ and the canonical dual pairing $\langle\cdot, \cdot\rangle$; $\mathbb{B}_{X}$ stands for the closed unit ball in $X$ (we use the simplified notation $\mathbb{B}$ and $\mathbb{B}^{*}$ for the dual balls in $X$ and $X^{*}$ if no confusion arises). Given spaces $X$ and $Y, \mathscr{B}(X, Y)$ denotes the collection of all bounded linear operators from $X$ to $Y$.

Let $L \subset X$ be a closed subspace of $X$. A projection from $X$ to $L$ is an operator $\pi_{L} \in$ $\mathscr{B}(X, X)$ such that the image of $X$ under $\pi_{L}$ is $L$ and the restriction of $\pi_{L}$ on $L$ is the identity operator. We will drop the subindex $L$ if there is no confusion. Recall that $L$ is complemented in $X$ if there is a closed subspace $M$ of $X$ with $X=L \oplus M$. It is well known that $L$ is complemented in $X$ if and only if there is a projection from $X$ to $L$, which happens, in particular, when $L$ is of finite dimension or finite codimension. Note also that every closed subspace of $X$ is complemented in $X$ if and only if $X$ is isomorphic to a Hilbert space.

2. Restrictive metric regularity. Recall that metric regularity is a concept defined for mappings between metric spaces; see [11] and the references therein. Given two metric spaces $\left(E_{1}, d_{1}\right)$ and $\left(E_{2}, d_{2}\right)$ and a mapping $f: E_{1} \rightarrow E_{2}$, we say that $f$ is metrically regular around $\bar{x}$ if there are neighborhoods $U$ of $\bar{x}$ and $V$ of $f(\bar{x})$ and a constant $\mu>0$ such that

$$
\operatorname{dist}\left(x ; f^{-1}(y)\right):=\inf _{v \in f^{-1}(y)} d_{1}(x, v) \leq \mu d_{2}(f(x), y) \quad \forall x \in U, y \in V .
$$

When both spaces $E_{i}$ in (2.1) are Banach (with $X:=E_{1}, Y:=E_{2}$, and the same notation $\|\cdot\|$ for the norms on $X$ and $Y$ ), (2.1) obviously reduces to (1.1). If in this case, $f: X \rightarrow Y$ is strictly differentiable at $\bar{x}$, then the surjectivity of $\nabla f(\bar{x}): X \rightarrow Y$ is necessary and sufficient for the metric regularity of $f$ around $\bar{x}$. What could be said about metric regularity of $f$ when $\nabla f(\bar{x})$ is not surjective? We suggest to consider the following property concerning metric regularity of the restrictive mapping $f: X \rightarrow f(X)$.

DEFINITION 2.1. Let $f: X \rightarrow Y$ be a mapping between Banach spaces. $f$ is said to have the RMR property around $\bar{x}$, or $f$ is RMR around this point, if the restrictive mapping $f: X \rightarrow f(X)$ between $X$ and the metric space $f(X) \subset Y$, whose metric is induced by the norm on $Y$, is metrically regular around $\bar{X}$ in the sense of (2.1).

One can easily see, by the classical open mapping theorem, that for linear mappings $f$, the RMR property always holds if the subspace $f(X)$ is closed in $Y$. However, the situation is much more complicated for nonlinear mappings when the RMR property may be violated even in the simplest cases as, for example, for $f(x)=x^{2}$ around $\bar{x}=$ $0 \in \mathbb{R}$. 
Note that, although metric regularity is a local property, the image $f(X)$ is a nonlocal object in the sense that some points $x$ situated far from $\bar{x}$ may contribute to the image of $f$ around $f(\bar{x})$. Indeed, consider the mapping $f: \mathbb{R}^{2} \rightarrow \mathbb{R}^{2}$ defined by

$$
f(x):= \begin{cases}\left(x_{1}-1,0\right) & \text { if } x_{1}>1 \\ \left(x_{1}, x_{1}^{2}\right) & \text { otherwise }\end{cases}
$$

for all $\left(x_{1}, x_{2}\right) \in \mathbb{R}^{2}$. Then $f$ is not $\mathrm{RMR}$ around $(0,0)$, while the localized mapping $f: \mathbb{B}_{\mathbb{R}^{2}} \rightarrow f\left(\mathbb{B}_{\mathbb{R}^{2}}\right)$ satisfies the metric regularity property (2.1) around this point. Thus it might be more appropriate to consider the metric regularity of $f: U \rightarrow f(U)$ for some neighborhood $U$ of $\bar{x}$. However, the latter property is obviously equivalent to RMR of the modified mapping $\tilde{f}: X \rightarrow Y$ defined by

$$
\tilde{f}(x):= \begin{cases}f(x) & \text { if } x \in U, \\ \tilde{y} & \text { otherwise }\end{cases}
$$

where $\tilde{y}$ is any fixed point of $Y$ different from $f(\bar{x})$. This allows us to confine our consideration to the RMR property introduced above.

In the remaining part of this section, we establish effective necessary conditions, sufficient conditions, and characterizations for the RMR property of mappings between general Banach spaces. We start with an important necessary condition, which is widely used in what follows.

THEOREM 2.2. Let $X$ and $Y$ be Banach spaces, and let $f: X \rightarrow Y$ be RMR around $\bar{x}$ and Fréchet differentiable at this point. Then $\nabla f(\bar{x})(X)$ is closed in $Y$.

Proof. Choose $\delta>0$ such that for any $x \in \bar{x}+\delta \mathbb{B}$, there is $\tilde{x} \in f^{-1}(f(x))$ satisfying $\|\tilde{x}-\bar{x}\| \leq \mu\left\|f(x)-f\left(x_{0}\right)\right\|$ for some constant $\mu>0$. Let $y_{0} \in \operatorname{cl} A(X)$ for $A:=\nabla f(\bar{x})$. Then there are $y_{k} \rightarrow y_{0}$ with $y_{k} \in A(X)$ and such that $\left\|y_{k+1}-y_{k}\right\|<1 / 2^{k}$ for all $k \in$ $\mathbb{N}:=\{1,2, \ldots\}$. To proceed, we build a sequence $\left\{x_{k}\right\} \subset X$ with the following properties:

$$
\left\|x_{k+1}-x_{k}\right\|<\frac{3 \mu}{2^{k}}, \quad\left\|y_{k}-A\left(x_{k}\right)\right\|<\frac{1}{2^{k}}, \quad k \in \mathbb{N} .
$$

Define $x_{k}$ iteratively. First let $x_{1}$ be any point with $A\left(x_{1}\right)=y_{1}$. Then having $x_{1}, \ldots, x_{k}$ that satisfy (2.4), define $x_{k+1}$ as follows. Fix $u \in \in^{-1}\left(y_{k+1}\right)-x_{k}$ and choose a small $t>0$ such that $t\|u\|<\delta$ and

$$
\left\|\frac{f(\bar{x}+t h)-f(\bar{x})}{t}-A(h)\right\|<\frac{1}{2^{k+2}} \quad \text { whenever } h \in \max \left\{\|u\|, \frac{3 \mu}{2^{k}}\right\} \mathbb{B} .
$$

This gives, in particular, that

$$
\left\|\frac{f(\bar{x}+t u)-f(\bar{x})}{t}-A(u)\right\|<\frac{1}{2^{k+2}},
$$


which implies the estimates

$$
\begin{aligned}
\|f(\bar{x}+t u)-f(\bar{x})\| & <t\left(\|A(u)\|+\frac{1}{2^{k+2}}\right) \\
& =t\left(\left\|y_{k+1}-A\left(x_{k}\right)\right\|+\frac{1}{2^{k+2}}\right) \\
& \leq t\left(\left\|y_{k+1}-y_{k}\right\|+\left\|y_{k}-A\left(x_{k}\right)\right\|+\frac{1}{2^{k+2}}\right) \\
& <t\left(\frac{1}{2^{k}}+\frac{1}{2^{k}}+\frac{1}{2^{k+2}}\right)<\frac{3 t}{2^{k}} .
\end{aligned}
$$

Using now the RMR property of $f$ around $\bar{x}$, select $x^{\prime} \in X$ with $f\left(x^{\prime}\right)=f(\bar{x}+t u)$ and

$$
\left\|x^{\prime}-\bar{x}\right\|<\frac{3 \mu t}{2^{k}}
$$

Putting $v:=\left(x^{\prime}-\bar{x}\right) / t$ and $x_{k+1}:=x_{k}+v$, we observe that $x_{k}, x_{k+1}$ satisfy the first inequality in (2.4). It remains to show that

$$
\left\|y_{k+1}-A\left(x_{k+1}\right)\right\|<\frac{1}{2^{k+1}} .
$$

To furnish this, note that

$$
\left\|\frac{f(\bar{x}+t v)-f(\bar{x})}{t}-A(v)\right\|<\frac{1}{2^{k+2}}
$$

by (2.5). Thus one has the estimate

$$
\left\|\frac{f(\bar{x}+t u)-f(\bar{x})}{t}-A(v)\right\|<\frac{1}{2^{k+2}}
$$

due to $\bar{x}+t v=x^{\prime}$ and $f\left(x^{\prime}\right)=f(\bar{x}+t u)$. Combining the latter inequality with that in (2.6), we arrive at

$$
\|A(u)-A(v)\|<\frac{1}{2^{k+1}} .
$$

Then the required estimate (2.9) follows from the observation that $A(u)-A(v)=y_{k+1}-$ $A\left(x_{k+1}\right)$, which justifies (2.4).

It is clear from (2.4) that $\left\{x_{k}\right\}$ is a Cauchy sequence in $X$, and hence it converges to some point $\hat{x} \in X$. On the other hand, we have from (2.4) that $\lim A\left(x_{k}\right)=\lim y_{k}=y_{0}$. Thus $A(\hat{x})=y_{0}$, that is, $y_{0} \in A(X)$. By the choice of $y_{0}$, we finally conclude that $A(X)$ is closed in $Y$, which completes the proof of the theorem.

REMARK 2.3. It is easy to observe from the proof of Theorem 2.2 that the same conclusion holds true if the RMR property of $f$ around $\bar{x}$ is relaxed as follows: there are a neighborhood $V$ of $f(\bar{x})$ and a constant $\mu>0$ such that, given any $y \in V \cap f(X)$, there exists $x^{\prime} \in f^{-1}(y)$ satisfying $\left\|x^{\prime}-\bar{x}\right\|<\mu\|y-f(\bar{x})\|$. Also we do not need to require the completeness of the normed space $Y$. Indeed, a slight modification of the proof allows us to show that $A(X)$ is complete in this case, which is all we need. 
To continue, for the given mapping $f: X \rightarrow Y$, we define the Lipschitzian modulus of $f$ in the ball $\bar{x}+\delta \mathbb{B}_{X}$ by

$$
\ell_{f}(\bar{x} ; \delta):=\sup _{\substack{x_{1}, x_{2} \in \bar{x}+\delta \mathbb{B}_{X} \\ x_{1} \neq x_{2}}} \frac{\left\|f\left(x_{1}\right)-f\left(x_{2}\right)\right\|}{\left\|x_{1}-x_{2}\right\|}
$$

and observe that $f$ is Lipschitz continuous around $\bar{x}$ if and only if $\ell_{f}(\bar{x} ; \delta)<\infty$ for some $\delta>0$. Given another mapping $g: X \rightarrow Y$, we denote for simplicity $\ell_{f, g}(\bar{x} ; \delta):=$ $\ell_{f-g}(\bar{x} ; \delta)$. In this notation, a mapping $f: X \rightarrow Y$ is strictly differentiable at $\bar{x}$ if there is a bounded linear operator $\nabla f(\bar{x}): X \rightarrow Y$ with $\ell_{f, \nabla f(\bar{x})}(\bar{x} ; \delta) \rightarrow 0$ as $\delta \downarrow 0$. For convenience, we denote $r_{f}(\bar{x} ; \delta):=\ell_{f, \nabla f(\bar{x})}(\bar{x} ; \delta)$ and call the function $r_{f}(\bar{x} ; \cdot):(0, \infty) \rightarrow$ $(0, \infty]$ the rate of strict differentiability of $f$ at $\bar{x}$.

THeOREM 2.4. Let $X$ and $Y$ be Banach spaces, and let $A: X \rightarrow Y$ be a bounded linear operator such that the space $A(X)$ is closed and complemented in $Y$. Then there exist positive constants $\gamma$ and $\mu$ with the following properties.

Given $f: X \rightarrow Y$ with $\ell_{f, A}(\bar{x} ; \delta)<\gamma$ for some $\delta>0$, there are neighborhoods $U$ of $\bar{x}$ and $V$ of $f(\bar{x})$ such that for any $x \in U$ and $y \in V$, there is $x_{y} \in X$ satisfying the estimates

$$
\left\|y-f\left(x_{y}\right)\right\| \leq \mu \operatorname{dist}\left(y-f\left(x_{y}\right) ; A(X)\right), \quad\left\|x-x_{y}\right\| \leq \mu\|f(x)-y\| .
$$

To be precise, $x_{y}$ can be chosen so that the first estimate in (2.14) is replaced with

$$
\pi\left(y-f\left(x_{y}\right)\right)=0
$$

for any given projection $\pi$ from $Y$ to $A(X)$.

Proof. Since $A(X)$ is closed and complemented in $Y$, there is a closed subspace $Y_{1} \subset Y$ such that $Y=Y_{1} \oplus A(X)$. Picking any $y \in Y$, we uniquely represent it as $y=$ $y_{1}+y_{2}$ with some $y_{1} \in Y_{1}, y_{2} \in A(X)$ and define projections $\pi_{i}: Y \rightarrow Y$ by $\pi_{i}(y)=y_{i}$, $i=1,2$. It is well known that the norm

$$
\|y\|_{1}:=\left\|\pi_{1}(y)\right\|+\left\|\pi_{2}(y)\right\|
$$

is equivalent to the original one. This gives us a constant $\mu_{1}>0$ with $\|y\|_{1} \leq \mu_{1}\|y\|$ for all $y \in Y$. Thus

$$
\begin{aligned}
\left\|\pi_{1}(y)\right\| & =\left\|\pi_{1}(y-\tilde{y})\right\| \leq\left\|\pi_{1}(y-\tilde{y})\right\|+\left\|\pi_{2}(y-\tilde{y})\right\| \\
& =\|y-\tilde{y}\|_{1} \leq \mu_{1}\|y-\tilde{y}\| \quad \forall y \in Y, \tilde{y} \in A(X) .
\end{aligned}
$$

Applying the classical open mapping theorem to the operator $A: X \rightarrow A(X)$, we find $\mu_{2}>0$ such that for any $y \in A(X)$, there is $x \in A^{-1}(y)$ with $\|x\| \leq \mu_{2}\|y\|$. Now denote

$$
\gamma:=\frac{1}{2 \mu_{1} \mu_{2}+2 \mu_{2}}, \quad \mu:=\max \left\{\mu_{1}, 2 \mu_{1} \mu_{2}\right\}
$$

and show that these are the constants we are looking for. 
It is clear that every $f$ satisfying the assumptions of the theorem is Lipschitz continuous around $\bar{x}$. Define

$$
V:=f(\bar{x})+\left(\frac{\delta}{8 \mu_{1} \mu_{2}}\right) \mathbb{B}, \quad U:=f^{-1}(V) \cap\left(\bar{x}+\left(\frac{\delta}{2}\right) \mathbb{B}\right)
$$

and fix $\hat{x} \in U, \tilde{y} \in V$. Starting with $x_{0}:=\hat{x}$, we find $x_{1} \in X$ such that

$$
\begin{gathered}
A\left(x_{1}-x_{0}\right)=\pi_{2}\left(\tilde{y}-f\left(x_{0}\right)\right), \\
\left\|x_{1}-x_{0}\right\| \leq \mu_{2}\left\|\pi_{2}\left(\tilde{y}-f\left(x_{0}\right)\right)\right\| \leq \mu_{1} \mu_{2}\|\tilde{y}-f(\hat{x})\| \leq \frac{\delta}{4},
\end{gathered}
$$

which implies $x_{1} \in \bar{x}+\delta \mathbb{B}$. Next we iteratively define a sequence $\left\{x_{k}\right\} \subset X$ as follows. Given $x_{k}, x_{k+1}$, choose $x_{k+2}$ satisfying

$$
\begin{gathered}
A\left(x_{k+2}-x_{k+1}\right)=A\left(x_{k+1}-x_{k}\right)-\pi_{2}\left(f\left(x_{k+1}\right)-f\left(x_{k}\right)\right), \\
\left\|x_{k+2}-x_{k+1}\right\| \leq \mu_{2}\left\|A\left(x_{k+1}-x_{k}\right)-\pi_{2}\left(f\left(x_{k+1}\right)-f\left(x_{k}\right)\right)\right\| .
\end{gathered}
$$

We proceed to show that $x_{k} \in \bar{x}+\delta \mathbb{B}$ by induction. Assume $x_{0}, \ldots, x_{k+1} \in \bar{x}+\delta \mathbb{B}$ and get

$$
\begin{aligned}
\left\|x_{k+2}-x_{k+1}\right\| & \leq \mu_{2}\left\|A\left(x_{k+1}-x_{k}\right)-\pi_{2}\left(f\left(x_{k+1}\right)-f\left(x_{k}\right)\right)\right\| \\
& =\mu_{2}\left\|-\left[f\left(x_{k+1}\right)-f\left(x_{k}\right)-A\left(x_{k+1}-x_{k}\right)\right]+\pi_{1}\left(f\left(x_{k+1}\right)-f\left(x_{k}\right)\right)\right\| \\
& \leq \mu_{2}\left(\left\|f\left(x_{k+1}\right)-f\left(x_{k}\right)-A\left(x_{k+1}-x_{k}\right)\right\|+\left\|\pi_{1}\left(f\left(x_{k+1}\right)-f\left(x_{k}\right)\right)\right\|\right) \\
& \leq \mu_{2}\left(\ell_{f, A}(\bar{x} ; \delta)\left\|x_{k+1}-x_{k}\right\|+\mu_{1}\left\|f\left(x_{k+1}\right)-f\left(x_{k}\right)-A\left(x_{k+1}-x_{k}\right)\right\|\right) \\
& \leq \mu_{2}\left(1+\mu_{1}\right) \ell_{f, A}(\bar{x} ; \delta)\left\|x_{k+1}-x_{k}\right\|<\frac{1}{2}\left\|x_{k+1}-x_{k}\right\|,
\end{aligned}
$$

which clearly implies that

$$
\begin{gathered}
\left\|x_{k+2}-x_{k+1}\right\| \leq 2^{-k-1}\left\|x_{1}-x_{0}\right\|, \\
\left\|x_{k+2}-x_{0}\right\| \leq \sum_{i=0}^{k+1}\left\|x_{i+1}-x_{i}\right\| \leq \sum_{i=0}^{k+1} 2^{-i}\left\|x_{1}-x_{0}\right\|<2\left\|x_{1}-x_{0}\right\| \leq \frac{\delta}{2} .
\end{gathered}
$$

Hence $x_{k+2} \in \bar{x}+\delta \mathbb{B}$, and (2.23) holds for all $k \geq 0$ by induction. The latter implies that for any $m \in \mathbb{N}$, one has

$$
\left\|x_{k+m}-x_{k}\right\| \leq \sum_{i=0}^{m-1}\left\|x_{k+i+1}-x_{k+i}\right\| \leq \sum_{i=0}^{m-1} 2^{-k-i}\left\|x_{1}-x_{0}\right\| \longrightarrow 0 \quad \text { as } k \longrightarrow \infty
$$

Therefore, $\left\{x_{k}\right\}$ is a Cauchy sequence that converges to some point $\tilde{x} \in X$. By (2.24), we observe that

$$
\|\tilde{x}-\hat{x}\| \leq 2\left\|x_{1}-x_{0}\right\| \leq 2 \mu_{1} \mu_{2}\|\tilde{y}-f(\hat{x})\| \leq \mu\|\tilde{y}-f(\hat{x})\|,
$$


which ensures the second estimate in (2.14). It remains to show that $\tilde{y}-f(\tilde{x}) \in Y_{1}$ which gives (2.15) and hence the first estimate in (2.14) by (2.17). It follows from (2.20) and (2.21) that

$$
A\left(x_{k+2}-x_{k+1}\right)=-\pi_{2}\left(f\left(x_{k+1}\right)-\tilde{y}\right) \quad \forall k \in \mathbb{N} .
$$

Passing there to the limit as $k \rightarrow \infty$, we get $\pi_{2}(f(\tilde{x})-\tilde{y})=0$ and complete the proof.

The proof of Theorem 2.4 is based on a modified iteration procedure that goes back to the original proofs of Lyusternik and Graves. The above result, in contrast to other generalizations of the Lyusternik-Graves theorem, does not require the surjectivity assumption on underlying linear operator $A$, while it requires that $A(X)$ be complemented in $Y$. The latter condition is essential for property (2.15). We strongly believe that it can be relaxed to establish the metric regularity estimates (2.14).

When $A$ is surjective, Theorem 2.4 implies the following well-known result that was mainly obtained by Ioffe and Tihomirov [12] with a different proof; see also [6] and the references therein.

Corollary 2.5. Let $A: X \rightarrow Y$ be a surjective bounded linear operator between Banach spaces. Then there is $\gamma>0$ such that every mapping $f: X \rightarrow Y$ with $\ell_{f, A}(\bar{x} ; \delta)<\gamma$ for some $\delta>0$ is metrically regular around $\bar{x}$ and the constant $\mu>0$ in the metric regularity property can be chosen independent of $f$.

When $A$ is injective in Theorem 2.4, we have the next corollary ensuring the uniform RMR property of $f$ around $\bar{x}$.

Corollary 2.6. Let $A: X \rightarrow Y$ be an injective bounded linear operator between $\mathrm{Ba}-$ nach spaces. Assume that $A(X)$ is closed and complemented in $Y$. Then there is $\gamma>0$ such that, for every mapping $f: X \rightarrow Y$ satisfying $\ell_{f, A}(\bar{x} ; \delta)<\gamma$ with some number $\delta>0$, the localized mapping $f:[\bar{x}+\delta \mathbb{B}] \rightarrow f(\bar{x}+\delta \mathbb{B})$ is metrically regular around $\bar{x}$ with a constant $\mu>0$ independent of $f$.

Proof. Consider the mapping $\tilde{f}:=(\pi \circ f): X \rightarrow A(X)$, where $\pi$ is a projection from $Y$ to $A(X)$. Using Corollary 2.5, we conclude that $\tilde{f}$ is open around $\bar{x}$. Since the linear operator $A: X \rightarrow A(X)$ is surjective and injective, its inverse operator $A^{-1}$ : $A(X) \rightarrow X$ is single-valued and bounded. We show that $\tilde{f}$ is injective on $\bar{x}+\delta \mathbb{B}$ when $\gamma<\|\pi\|^{-1}\left\|A^{-1}\right\|^{-1}$. Indeed, if $\tilde{f}\left(x_{1}\right)=\tilde{f}\left(x_{2}\right)$ for $x_{1}, x_{2} \in \bar{x}+\delta \mathbb{B}$ with $x_{1} \neq x_{2}$, then

$$
\begin{aligned}
& \frac{\left\|f\left(x_{1}\right)-f\left(x_{2}\right)-A\left(x_{1}-x_{2}\right)\right\|}{\left\|x_{1}-x_{2}\right\|} \\
& \quad \geq \frac{\|\pi\|^{-1}\left\|\tilde{f}\left(x_{1}\right)-\tilde{f}\left(x_{2}\right)-A\left(x_{1}-x_{2}\right)\right\|}{\left\|x_{1}-x_{2}\right\|} \\
& \quad=\frac{\|\pi\|^{-1}\left\|A\left(x_{1}-x_{2}\right)\right\|}{\left\|x_{1}-x_{2}\right\|} \geq\|\pi\|^{-1}\left\|A^{-1}\right\|^{-1},
\end{aligned}
$$

which contradicts the choice of $\gamma$. Then we apply Theorem 2.4 to get the conclusion. 
For further results, we need to introduce another modulus involving the mapping $f: X \rightarrow Y$ and a linear operator $A: X \rightarrow Y$ between Banach spaces:

$$
\vartheta_{f, A}(\bar{x} ; \delta):=\sup _{y_{1}, y_{2} \in f(X) \cap(f(\bar{x})+\delta \mathbb{B})} \frac{\operatorname{dist}\left(y_{2}-y_{1} ; A(X)\right)}{\left\|y_{2}-y_{1}\right\|} .
$$

The following useful relationship can be easily derived from the definitions.

Proposition 2.7. Taking a mapping $f: X \rightarrow Y$ and a linear operator $A: X \rightarrow Y$ between Banach spaces, assume that $f$ is $R M R$ around $\bar{x}$. Then there are positive numbers $\mu>0$ and $\delta_{0}$ such that

$$
\vartheta_{f, A}(\bar{x} ; \delta) \leq \mu \ell_{f, A}(\bar{x} ; \mu \delta) \quad \forall \delta<\delta_{0} .
$$

The next result shows that, roughly speaking, $f: X \rightarrow f(X) \subset Y$ is RMR around $\bar{x}$ if and only if $f(X)$ is locally homeomorphic to $A(X)$ for some linear bounded operator $A: X \rightarrow Y$ close to $f$, that is, the modulus $\ell_{f, A}$ is sufficiently small.

Proposition 2.8. Let $A: X \rightarrow Y$ be a linear bounded operator between Banach spaces such that $A(X)$ is closed and complemented in $Y$, and let $\pi$ be a projection from $Y$ to $A(X)$. Then the following hold.

(a) There is $\gamma>0$ such that, for every mapping $f: X \rightarrow Y$ satisfying the RMR property around $\bar{x}$ and the estimate $\ell_{f, A}(\bar{x} ; \delta)<\gamma$ with some $\delta>0$, the projection $\pi$ is a local homeomorphism between $f(X)$ and $A(X)$ around $f(\bar{x})$.

(b) There is $\gamma>0$ such that every mapping $f: X \rightarrow Y$ satisfying $\ell_{f, A}(\bar{x} ; \delta)<\gamma$ with some $\delta>0$ is RMR around $\bar{x}$ provided that the projection $\pi$ is a local homeomorphism between $f(X)$ and $A(X)$ around $f(\bar{x})$.

Proof. It follows from Theorem 2.4 and Proposition 2.7.

COROLLARY 2.9. Let $f: X \rightarrow Y$ be a mapping between Banach spaces that is strictly differentiable at $\bar{x}$. Assume that $\nabla f(\bar{x})(X)$ is closed and complemented in $Y$. Then $f$ is $R M R$ around $\bar{x}$ if and only if each projection from $Y$ to $\nabla f(\bar{x})(X)$ is a local homeomorphism between $f(X)$ and $\nabla f(\bar{x})(X)$.

The next result gives necessary and sufficient conditions for RMR of strictly differentiable mappings. In its formulation, we use, beside the modulus $\vartheta$ from (2.29), the following construction of the tangent cone to a set $\Omega \subset X$ at $\bar{x} \in \Omega$ :

$$
T(\bar{x} ; \Omega):=\left\{v \in X \mid \exists v_{k} \longrightarrow v, t_{k} \downarrow 0, x_{k} \stackrel{\Omega}{\longrightarrow} \bar{x} \text { with } x_{k}+t_{k} v_{k} \in \Omega\right\},
$$

where $x_{k} \stackrel{\Omega}{\rightarrow} \bar{x}$ means that $x_{k} \rightarrow \bar{x}$ with $x_{k} \in \Omega$ as $k \rightarrow \infty$. Note that this tangent cone is an enlargement of the well-known (Bouligand) contingent cone corresponding to (2.29) with $x_{k}=\bar{x}$ for all $k \in \mathbb{N}$. 
THEOREM 2.10. Let $f: X \rightarrow Y$ be a mapping between Banach spaces that is strictly differentiable at $\bar{x}$. Consider the following conditions:

(a) $f$ is $R M R$ around $\bar{x}$;

(b) $\vartheta_{f, \nabla f(\bar{x})}(\bar{x} ; \delta) \rightarrow 0$ as $\delta \downarrow 0$ and $\nabla f(\bar{x})(X)$ is closed;

(c) $T(f(\bar{x}) ; f(X))=\nabla f(\bar{x})(X)$.

Then $(a) \Rightarrow(b) \Rightarrow(c)$. Moreover, $(b) \Rightarrow(a)$ if $\nabla f(\bar{x})(X)$ is closed and complemented in $Y$. Also (c) $\Rightarrow$ (a) when $\operatorname{codim} \nabla f(\bar{x})(X)<\infty$.

Proof. Implication $(a) \Rightarrow(b)$ is straightforward, while $(b) \Rightarrow(a)$ follows from Proposition 2.8 under the assumptions made. To prove $(\mathrm{b}) \Rightarrow(\mathrm{c})$, we first observe that $\nabla f(\bar{x})(X)$ $\subset T(f(\bar{x}) ; f(X))$ by the strict differentiability of $f$ at $\bar{x}$. Now pick $u \in T(f(\bar{x}) ; f(X))$ and find by (2.31) sequences $u_{k} \rightarrow u, y_{k} \rightarrow f(\bar{x})$, and $t_{k} \downarrow 0$ such that $y_{k}+t_{k} u_{k} \in f(X)$ for all $k \in \mathbb{N}$. Property (b) ensures the existence of $\tilde{y}_{k} \in \nabla f(\bar{x})(X)$ with $\left\|u_{k}-\tilde{y}_{k} / t_{k}\right\| \rightarrow 0$ as $k \rightarrow \infty$. This gives $\tilde{y}_{k} / t_{k} \rightarrow u$ as $k \rightarrow \infty$ and hence $u \in \nabla f(\bar{x})(X)$, which yields (c).

Finally, we prove $(\mathrm{c}) \Rightarrow(\mathrm{a})$ assuming that the space $\nabla f(\bar{x})(X)$ is finite codimensional. Thus there is a finite-dimensional space $Y_{1} \subset Y$ with $Y_{1} \oplus \nabla f(\bar{x})(X)=Y$. By Theorem 2.4, we find neighborhoods $U$ of $\bar{x}$ and $V$ of $f(\bar{x})$ as well as a number $\mu>0$ such that, for any $x \in U$ and $y \in V$, there exists $x_{y} \in X$ satisfying

$$
y-f\left(x_{y}\right) \in Y_{1}, \quad\left\|x-x_{y}\right\| \leq \mu\|f(x)-y\| .
$$

To justify (a), one needs to show that $y=f\left(x_{y}\right)$ provided that $y \in f(X)$ and that $U$ and $V$ are sufficiently small. Assuming the contrary, we find $x_{k} \rightarrow \bar{x}$ and $y_{k} \rightarrow f(\bar{x})$ with $y_{k} \in f(X)$ such that $0 \neq y_{k}-f\left(x_{k}\right) \in Y_{1}$. Denote $t_{k}:=\left\|y_{k}-f\left(x_{k}\right)\right\|$ and $u_{k}:=$ $\left(y_{k}-f\left(x_{k}\right)\right) / t_{k}$. Since $Y_{1}$ is finite dimensional, $u_{k}$ converges to some $0 \neq u \in Y_{1}$ along a subsequence. On the other hand, $u \in T(f(\bar{x}) ; f(X))=\nabla f(\bar{x})(X)$ by (c) and the construction of $u_{k}$. Thus $u=0$, which is a contradiction. This completes the proof of (c) $\Rightarrow$ (a) and of the whole theorem.

3. First-order calculus. In this section, we give applications of the RMR property to first-order calculus rules for sequential limiting generalized differential constructions in arbitrary Banach spaces. Other applications of metric regularity and related properties to generalized differential calculus can be found in $[11,13,15]$, and the references therein. The results presented below seem to be new even in the case of applications of the classical (not restrictive) metric regularity property for mappings between general Banach spaces.

First we define the generalized differential constructions of our study; see [19, 25] and their bibliographies for the history of these constructions and more discussions.

Given $\Omega \subset X$ and $\bar{x} \in \Omega$, we define the set of $\varepsilon$-normals to $\Omega$ at $\bar{x}$ by

$$
\hat{N}_{\varepsilon}(\bar{x} ; \Omega):=\left\{x^{*} \in X^{*} \mid \limsup _{x \stackrel{\Omega}{\rightarrow} \bar{x}} \frac{\left\langle x^{*}, x-\bar{x}\right\rangle}{\|x-\bar{x}\|} \leq \varepsilon\right\}, \quad \varepsilon \geq 0 .
$$


When $\varepsilon=0$, this is a cone called the prenormal cone or the Fréchet normal cone and denoted by $\hat{N}(\bar{x} ; \Omega)$. Then the basic/limiting normal cone to $\Omega$ at $\bar{x}$ is given by

$$
N(\bar{x} ; \Omega):=\left\{x^{*} \in X^{*} \mid \exists \varepsilon_{k} \downarrow 0, x_{k}^{*} \stackrel{w^{*}}{\longrightarrow} x^{*}, x_{k} \stackrel{\Omega}{\longrightarrow} \bar{x} \text { with } x_{k}^{*} \in \hat{N}_{\varepsilon_{k}}\left(x_{k} ; \Omega\right)\right\},
$$

where the limits in (3.2) are sequential. When $X$ is Asplund and $\Omega$ is closed around $\bar{x}$, one can equivalently put $\varepsilon_{k}=0$ in (3.2) and the subsequent limiting constructions; see [25]. However, $\varepsilon_{k}$ cannot be removed from the definitions without loss of crucial properties in general Banach space settings, as one can see in the arguments and results below.

A set $\Omega \subset X$ is called normally regular at $\bar{x} \in \Omega$ if $\hat{N}(\bar{x} ; \Omega)=N(\bar{x} ; \Omega)$. This class includes, in particular, all convex sets, sets with smooth boundaries, and so forth. New calculus results for normal regularity are obtained in this section being incorporated in calculus rules for normal cones.

Given a set-valued mapping $F: X \rightrightarrows Y$ between Banach spaces, we define its $\varepsilon$ coderivative, normal coderivative, and mixed coderivative at $(\bar{x}, \bar{y}) \in \operatorname{gph} F$ by, respectively,

$$
\begin{aligned}
\hat{D}_{\varepsilon}^{*} F(\bar{x}, \bar{y})\left(y^{*}\right):= & \left\{x^{*} \in X^{*} \mid\left(x^{*},-y^{*}\right) \in \hat{N}_{\varepsilon}((\bar{x}, \bar{y}) ; \operatorname{gph} F)\right\}, \\
D_{N}^{*} F(\bar{x}, \bar{y})\left(y^{*}\right):= & \left\{x^{*} \in X^{*} \mid\left(x^{*},-y^{*}\right) \in N((\bar{x}, \bar{y}) ; \operatorname{gph} F)\right\}, \\
D_{M}^{*} F(\bar{x}, \bar{y})\left(y^{*}\right):= & \left\{x^{*} \in X^{*} \mid \exists \varepsilon_{k} \downarrow 0,\left(x_{k}, y_{k}\right) \stackrel{F}{\longrightarrow}(\bar{x}, \bar{y}),\right. \\
& \left.x_{k}^{*} \stackrel{w^{*}}{\longrightarrow} x^{*}, y_{k}^{*} \stackrel{\|\cdot\|}{\longrightarrow} y^{*}, \text { with } x_{k}^{*} \in \hat{D}_{\varepsilon_{k}}^{*} F\left(x_{k}, y_{k}\right)\left(y_{k}^{*}\right)\right\},
\end{aligned}
$$

for all $y^{*} \in Y^{*}$, where $\left(x_{k}, y_{k}\right) \stackrel{F}{\rightarrow}(\bar{x}, \bar{y})$ means that $\left(x_{k}, y_{k}\right) \rightarrow(\bar{x}, \bar{y})$ with $\left(x_{k}, y_{k}\right) \in$ $\operatorname{gph} F$. We say that $F$ is $N$-regular (resp., $M$-regular) at $(\bar{x}, \bar{y})$ if $\hat{D}^{*} F(\bar{x}, \bar{y})=D_{N}^{*} F(\bar{x}, \bar{y})$ (resp., $\hat{D}^{*} F(\bar{x}, \bar{y})=D_{M}^{*} F(\bar{x}, \bar{y})$ ).

Given an extended real-valued function $\varphi: X \rightarrow \mathbb{R}:=[-\infty, \infty]$ finite at $\bar{x}$, we define its (first-order) $\varepsilon$-subdifferential and basic subdifferential by, respectively,

$$
\widehat{\partial}_{\varepsilon} \varphi(\bar{x}):=\hat{D}_{\varepsilon}^{*} E_{\varphi}(\bar{x}, \varphi(\bar{x}))(1), \quad \partial \varphi(\bar{x}):=D^{*} E_{\varphi}(\bar{x}, \varphi(\bar{x}))(1),
$$

where $E_{\varphi}: X \rightarrow \mathbb{R}$ is the epigraphical multifunction with $\operatorname{gph} E_{\varphi}=\operatorname{epi} \varphi$ and where $D^{*} E_{\varphi}:=D_{N}^{*} E_{\varphi}=D_{M}^{*} E_{\varphi}$, since there is no difference between the normal and mixed coderivatives for mappings with values in finite-dimensional spaces. A function $\varphi$ is said to be lower regular at $\bar{x}$ if $\hat{\partial} \varphi(\bar{x})=\partial \varphi(\bar{x})$.

Note that the above subdifferential constructions admit intrinsic analytic representations not directly involving generalized normals; see [25] and its references. The given definitions allow us to develop a geometric approach to generalized differential calculus based just on calculus rules for generalized normals.

First we obtain two-sided uniform estimates of $\varepsilon$-normals for inverse images of sets

$$
f^{-1}(\Omega):=\{x \in X \mid f(x) \in \Omega\}
$$

under mappings $f: X \rightarrow Y$ having the RMR property. 
THEOREM 3.1. Let $f: X \rightarrow Y$ be a mapping between Banach spaces that is Lipschitz continuous around some point $\bar{x}$, let $A: X \rightarrow Y$ be a linear bounded operator, and let $\Omega$ be a subset of $Y$ with $\bar{y}:=f(\bar{x}) \in \Omega$. Then there are positive constants $\bar{\delta}$ and $\mu_{1}$ such that for every $\varepsilon \geq 0, \delta \in(0, \bar{\delta}), \hat{x} \in f^{-1}(\Omega) \cap(\bar{x}+\delta \mathbb{B})$, and $y^{*} \in \hat{N}_{\varepsilon}(f(\hat{x}) ; \Omega \cap f(X))$,

$$
A^{*} y^{*} \in \hat{N}_{\varepsilon_{1}}\left(\hat{x} ; f^{-1}(\Omega)\right) \quad \varepsilon_{1}:=\mu_{1} \varepsilon+\left\|y^{*}\right\| \ell_{f, A}(\bar{x} ; \delta)
$$

If, in addition, $f$ is $R M R$ around $\bar{x}$ and $A(X)$ is closed in $Y$, then there is $\mu_{2}>0$ such that for every $x^{*} \in \hat{N}_{\varepsilon}\left(\hat{x} ; f^{-1}(\Omega)\right), \hat{x}^{*} \in x^{*}+\left(\varepsilon+\mu_{2}\left(\varepsilon+\left\|x^{*}\right\|\right) \ell_{f, A}(\bar{x} ; \delta)\right) \mathbb{B}^{*}$ satisfying $\left(A^{*}\right)^{-1}\left(\hat{x}^{*}\right) \neq \varnothing$ and

$$
y^{*} \in \widehat{N}_{\varepsilon_{2}}(f(\hat{x}) ; \Omega \cap f(X)) \quad \varepsilon_{2}:=\mu_{2} \varepsilon+\mu_{2}\left(\left\|x^{*}\right\|+\left\|y^{*}\right\|\right) \ell_{f, A}(\bar{x} ; \delta)
$$

whenever $y^{*} \in\left(A^{*}\right)^{-1}\left(\hat{x}^{*}\right)$.

Proof. Without loss of generality, we assume that $\Omega \subset f(X)$ throughout the proof. Fix $\hat{x}$ as in the theorem and put $\hat{y}:=f(\hat{x}), y^{*} \in \hat{N}_{\varepsilon}(\hat{y} ; \Omega)$. Choose $\bar{\delta}$ such that $\ell_{f}(\bar{x} ; \bar{\delta})<$ $\infty$. Then we have

$$
\begin{aligned}
\limsup _{x \stackrel{f^{-1}(\Omega)}{\longrightarrow} \hat{x}} \frac{\left\langle A^{*} y^{*}, x-\hat{x}\right\rangle}{\|x-\hat{x}\|}= & \limsup \frac{\left\langle y^{*}, A(x-\hat{x})\right\rangle}{\|x-\hat{x}\|} \\
\leq & \limsup ^{f^{-1}(\Omega)} \hat{x} \frac{\left\langle y^{*}, f(x)-f(\hat{x})\right\rangle}{\|x-\hat{x}\|}+\left\|y^{*}\right\| \ell_{f, A}(\bar{x} ; \delta) \\
& x \stackrel{f^{-1}(\Omega)}{\longrightarrow} \hat{x} \\
\leq & \limsup \max \left\{0, \frac{\left\langle y^{*}, y-\hat{y}\right\rangle}{\ell_{f}(\bar{x} ; \bar{\delta})^{-1}\|y-\hat{y}\|}\right\}+\left\|y^{*}\right\| \ell_{f, A}(\bar{x} ; \delta) \\
\leq & \ell_{f}(\bar{x} ; \bar{\delta}) \varepsilon+\left\|y^{*}\right\| \ell_{f, A}(\bar{x} ; \delta),
\end{aligned}
$$

which implies (3.8) with $\mu_{1}:=\ell_{f}(\bar{x} ; \bar{\delta})$ by definition (3.1).

The proof of (3.9) is more involved. First observe that

$$
\|f(\hat{x}+t v)-\hat{y}\| \leq \ell_{f, A}(\bar{x} ; \delta)\|v\| t
$$

for all $v \in \operatorname{ker} A$ and small $t>0$. By the RMR property of $f$ around $\bar{x}$, we find $x_{t} \in$ $f^{-1}(\hat{y})$ such that $\left\|\hat{x}+t v-x_{t}\right\| \leq \mu \ell_{f, A}(\bar{x} ; \delta)\|v\| t$ for some constant $\mu>0$ and for each small $t>0$. Then for any $\gamma>0$, one has

$$
\left\langle x^{*}, x_{t}-\hat{x}\right\rangle \leq(\varepsilon+\gamma)\left\|x_{t}-\hat{x}\right\| \leq(\varepsilon+\gamma)\left(1+\mu \ell_{f, A}(\bar{x} ; \delta)\right)\|v\| t
$$

when $t$ is close to zero. On the other hand,

$$
\left\langle x^{*}, x_{t}-\hat{x}\right\rangle=\left\langle x^{*}, t v\right\rangle+\left\langle x^{*}, x_{t}-\hat{x}-t v\right\rangle \geq t\left\langle x^{*}, v\right\rangle-\mu\left\|x^{*}\right\| \ell_{f, A}(\bar{x} ; \delta)\|v\| t .
$$


Thus we have the estimate

$$
\left\langle x^{*}, v\right\rangle \leq \mu\left\|x^{*}\right\| \ell_{f, A}(\bar{x} ; \delta)\|v\|+(\varepsilon+\gamma)\left(1+\mu \ell_{f, A}(\bar{x} ; \delta)\right)\|v\|
$$

for any $\gamma>0$, which implies that

$$
\left\langle x^{*}, v\right\rangle \leq \hat{\varepsilon}\|v\|
$$

whenever $v \in \operatorname{ker} A$, with

$$
\hat{\varepsilon}:=\varepsilon+\mu\left(\varepsilon+\left\|x^{*}\right\|\right) \ell_{f, A}(\bar{x} ; \delta) .
$$

The Hahn-Banach theorem allows us to extend $\left.x^{*}\right|_{\text {ker } A}$ to some $\tilde{x}^{*} \in X^{*}$ with $\left\|\tilde{x}^{*}\right\| \leq \hat{\varepsilon}$. Now we let $\hat{x}^{*}:=x^{*}-\tilde{x}^{*}$ and construct a linear functional $\hat{y}^{*}$ on $A(X)$ by

$$
\left\langle\hat{y}^{*}, y\right\rangle:=\left\langle\hat{x}^{*}, x\right\rangle \text { for any } y \in A(X), x \in A^{-1}(y) \text {. }
$$

Since $\operatorname{ker} A \subset \operatorname{ker}\left(\hat{x}^{*}\right)$, this functional is well defined. The RMR property of $A: X \rightarrow A(X)$ (which is automatic due to the closedness of $A(X)$ ) implies that $\hat{y}^{*}$ is bounded. Using the Hahn-Banach theorem again, we extend $\hat{y}^{*}$ to some functional $\tilde{y}^{*} \in Y^{*}$. One clearly has $\hat{x}^{*}=A^{*} \tilde{y}^{*}$, and hence $\left(A^{*}\right)^{-1}\left(\hat{x}^{*}\right) \neq \varnothing$.

Take an arbitrary functional $y^{*} \in\left(A^{*}\right)^{-1}\left(\hat{x}^{*}\right)$. It remains to show that $y^{*} \in \hat{N}_{\varepsilon_{2}}(\hat{y}$; $\Omega$ ), where $\varepsilon_{2}$ is defined in (3.9) with some $\mu_{2} \geq \mu$. To proceed, we use the assumed RMR property of $f$ around $\bar{x}$ and for any $y \in \Omega$ close to $\hat{y}$, find $x_{y} \in f^{-1}(y)$ such that $\left\|x_{y}-\hat{x}\right\| \leq \hat{\mu}\|y-\hat{y}\|$ with some constant $\hat{\mu}>0$. Therefore, $x_{y} \rightarrow \hat{x}$ when $y \rightarrow \hat{y}$. Furthermore,

$$
\left\|y-\hat{y}-A\left(x_{y}-\hat{x}\right)\right\| \leq \ell_{f, A}(\bar{x} ; \delta)\left\|x_{y}-\hat{x}\right\|
$$

whenever $y$ is close to $\hat{y}$. Thus we have the estimates

$$
\begin{aligned}
& \underset{y}{y \stackrel{\Omega}{\rightarrow} \hat{y}} \limsup \frac{\left\langle y^{*}, y-\hat{y}\right\rangle}{\|y-\hat{y}\|} \\
& \quad \leq \limsup _{y \stackrel{\Omega}{\rightarrow} \hat{y}}\left[\frac{\left\langle y^{*}, A\left(x_{y}-\hat{x}\right)\right\rangle}{\|y-\hat{y}\|}+\frac{\ell_{f, A}(\bar{x} ; \delta)\left\|x_{y}-\hat{x}\right\|}{\|y-\hat{y}\|}\left\|y^{*}\right\|\right] \\
& \leq \limsup _{y \stackrel{\Omega}{\rightarrow} \hat{y}} \frac{\left\langle x^{*}-\tilde{x}^{*}, x_{y}-\hat{x}\right\rangle}{\|y-\hat{y}\|}+\hat{\mu} \ell_{f, A}(\bar{x} ; \delta)\left\|y^{*}\right\| \\
& \leq \limsup _{y \stackrel{\Omega}{\rightarrow} \hat{y}}\left[\frac{\left\langle x^{*}, x_{y}-\hat{x}\right\rangle}{\|y-\hat{y}\|}+\frac{\left\|\tilde{x}^{*}\right\| \cdot\left\|x_{y}-\hat{x}\right\|}{\|y-\hat{y}\|}\right]+\hat{\mu} \ell_{f, A}(\bar{x} ; \delta)\left\|y^{*}\right\| \\
& \leq \limsup \max \left\{0, \frac{\left\langle x^{*}, x_{y}-\hat{x}\right\rangle}{\hat{\mu}^{-1}\left\|x_{y}-\hat{x}\right\|}\right\}+\hat{\mu} \hat{\varepsilon}+\hat{\mu} \ell_{f, A}(\bar{x} ; \delta)\left\|y^{*}\right\| \\
& \leq \hat{\mu} \varepsilon+\hat{\mu} \hat{\varepsilon}+\hat{\mu} \ell_{f, A}(\bar{x} ; \delta)\left\|y^{*}\right\| \leq \mu_{2} \varepsilon+\mu_{2}\left(\left\|x^{*}\right\|+\left\|y^{*}\right\|\right) \ell_{f, A}(\bar{x} ; \delta)=\varepsilon_{2},
\end{aligned}
$$

where $\mu_{2}:=\max \left\{\mu, \hat{\mu}+\hat{\mu} \mu \ell_{f, A}(\bar{x} ; \bar{\delta}), \hat{\mu} \mu, \hat{\mu}\right\} \geq \mu$. This completes the proof. 
REMARK 3.2. (i) When $f$ is strictly differentiable at $\bar{x}$, we can take $A:=\nabla f(\bar{x})$ with $\ell_{f, A}(\bar{x} ; \delta)$ replaced by the rate of strict differentiability $r_{f}(\bar{x} ; \delta)$ in Theorem 3.1. Note that the subspace $\nabla f(\bar{x})(X)$ is closed in $Y$ by Theorem 2.2. In this case, necessary and sufficient conditions for the RMR property of $f$ around $\bar{x}$ are given in Theorem 2.10. If, in addition, $\nabla f(\bar{x})$ is surjective, then $f$ is metrically regular around $\bar{x}$ by the LyusternikGraves theorem and (3.9) reduces to

$$
x^{*} \in \nabla f(\bar{x})^{*} \widehat{N}_{\varepsilon_{2}}(f(\hat{x}) ; \Omega)+\left(\varepsilon+\mu_{2}\left(\varepsilon+\left\|x^{*}\right\|\right) r_{f}(\bar{x} ; \eta)\right) \mathbb{B}^{*}
$$

for any $x^{*} \in \widehat{N}_{\varepsilon}\left(\hat{x} ; f^{-1}(\Omega)\right)$, where $\varepsilon_{2}:=\mu_{2} \varepsilon+\mu_{2}\left\|x^{*}\right\| r_{f}(\bar{x} ; \delta)$. In particular, when $\hat{x}$ is replaced by $\bar{x}$, the following holds:

$$
\widehat{N}_{\varepsilon}\left(\bar{x} ; f^{-1}(\Omega)\right) \subset \nabla f(\bar{x})^{*} \hat{N}_{\mu_{2} \varepsilon}(f(\bar{x}) ; \Omega)+\varepsilon \mathbb{B}^{*} \quad \forall \varepsilon \geq 0 .
$$

(ii) It is important to put $\Omega \cap f(X)$ in (3.9) but not $\Omega$. To illustrate this, consider $f: \mathbb{R}^{2} \rightarrow \mathbb{R}^{2}$ and $\Omega \subset \mathbb{R}^{2}$ defined by

$$
f(u, v):=(u, 0) \quad \forall(u, v) \in \mathbb{R}^{2}, \quad \Omega:=\left\{(u, v) \in \mathbb{R}^{2} \text { with }|u| \geq|v|\right\} .
$$

Then (3.9) fails for $\bar{x}=\hat{x}=0$ if $\Omega \cap f(X)$ is replaced by $\Omega$.

(iii) Note that the uniform estimates in Theorem 3.1 are distinguished from the results of "fuzzy calculus" type available under other assumptions for such constructions in Asplund spaces; see, for example, $[11,25]$ with their references. The main differences are that we get uniform qualitative estimates of $\varepsilon$-normals for all points around the reference ones, while fuzzy calculus results involve only some of them. These advantages of Theorem 3.1 are used in what follows.

Theorem 3.1 directly implies the following two calculus rules of equality type for Fréchet normals to inverse images.

COROLlary 3.3. Let $f: X \rightarrow Y$ be a mapping between Banach spaces that is strictly differentiable at $\bar{x}$ and such that $f(\bar{x}) \in \Omega$. Assume that $f$ is $R M R$ around $\bar{x}$. Then

$$
\begin{gathered}
\hat{N}\left(\bar{x} ; f^{-1}(\Omega)\right)=\nabla f(\bar{x})^{*} \hat{N}(f(\bar{x}) ; \Omega \cap f(X)), \\
\left(\nabla f(\bar{x})^{*}\right)^{-1} \hat{N}\left(\bar{x} ; f^{-1}(\Omega)\right)=\hat{N}(f(\bar{x}) ; \Omega \cap f(X)) .
\end{gathered}
$$

Proof. Both equalities follow from Theorem 3.1, with $A=\nabla f(\bar{x}), \varepsilon=0, \hat{x}=\bar{x}$, and $\delta \downarrow 0$.

Note that the first equality implies the second one in Corollary 3.3 when $\nabla f(\bar{x})$ is surjective, that is, when $f$ is metrically regular around $\bar{x}$. In general, they are independent as can be easily illustrated by simple examples.

Next, we intend to derive exact formulas for computing basic normals (3.2) to inverse images by passing to the limit from the estimates of Theorem 3.1. To proceed, we need to introduce first the following weak* extensibility property, which is related but somewhat different from the Banach extensibility property (see, e.g., [5]) and plays an essential role in the subsequent results of this paper. 
DEFINITION 3.4. Let $L$ be a closed linear subspace of a Banach space $X$. $L$ is $w^{*}$ extensible in $X$ if every sequence $\left\{v_{k}^{*}\right\} \subset L^{*}$, with $v_{k}^{*} \stackrel{w^{*}}{\longrightarrow} 0$ as $k \rightarrow \infty$, contains a subsequence $\left\{v_{k_{j}}^{*}\right\}$ such that each $v_{k_{j}}^{*}$ can be extended to $x_{j}^{*} \in X^{*}$ with $x_{j}^{*} \stackrel{w^{*}}{\longrightarrow} 0$ as $j \rightarrow \infty$.

The next proposition shows that the $w^{*}$-extensibility property always holds for complemented subspaces of arbitrary Banach spaces and also, unconditionally, in a broad class of Banach spaces including all Asplund spaces, weakly compactly generated spaces, spaces admitting smooth renorms of any kind, and so forth.

Proposition 3.5. Let $L$ be a closed linear subspace of a Banach space $X$. Then $L$ is $w^{*}$-extensible in $X$ if one of the following conditions holds:

(a) $L$ is complemented in $X$;

(b) the closed unit ball of $X^{*}$ is weak* sequentially compact.

Proof. Let $L$ be complemented in $X$, and let $\pi: X \rightarrow L$ be a projection operator. Putting $x_{k}^{*}:=\left\langle v_{k}^{*}, \pi(x)\right\rangle$ on $X$, we conclude that $x_{k}^{*}$ is an extension of $v_{k}^{*}$ with $x_{k}^{*} \stackrel{w^{*}}{\longrightarrow} 0$, that is, $L$ is $w^{*}$-extensible in $X$ in case (a).

To justify this property in case (b) for every $L \subset X$, we take an arbitrary sequence $v_{k}^{*}$ from Definition 3.4 and observe that it is bounded in $L^{*}$ due to the weak* convergence. By the Hahn-Banach theorem, we extend each $v_{k}^{*}$ to $\tilde{x}_{k}^{*} \in X^{*}$ such that the sequence $\left\{\tilde{x}_{k}^{*}\right\}$ is still bounded in $X^{*}$. Since $\mathbb{B}_{X^{*}}$ is assumed to be weak* sequentially compact, there are $x^{*} \in X^{*}$ and a weak* convergent subsequence $\tilde{x}_{k_{j}}^{*} \stackrel{w^{*}}{\longrightarrow} x^{*}$ as $j \rightarrow \infty$. Putting $x_{j}^{*}:=\tilde{x}_{k_{j}}^{*}-x^{*}$, we complete the proof of the proposition.

We show that the $w^{*}$-extensibility property may not hold even in some classical Banach spaces.

EXAMPLE 3.6. The subspace $L=c_{0}$ is not $w^{*}$-extensible in $X=\ell^{\infty}$.

Proof. Recall that $c_{0}$ is a Banach space of all real sequences convergent to zero that is endowed with the supremum norm. Let $v_{k}^{*}:=\xi_{k}^{*} \in c_{0}^{*}$, where $\xi_{k}^{*}$ maps every vector from $c_{0}$ to its $k$ th component. Assume that there is an increasing sequence of $k_{j} \in \mathbb{N}$ such that $v_{k_{j}}^{*}$ can be extended to $x_{j}^{*} \in\left(\ell^{\infty}\right)^{*}$ with $x_{j}^{*} \stackrel{w^{*}}{\longrightarrow} 0$. Define a closed linear subspace of $\ell^{\infty}$ by

$$
Z:=\left\{\left(\alpha_{1}, \alpha_{2}, \ldots\right) \in \ell^{\infty} \mid \alpha_{k}=0 \text { if } k \notin\left\{k_{1}, k_{2}, \ldots\right\}\right\}
$$

and a linear bounded operator $A: \ell^{\infty} \rightarrow Z$ by

$$
A\left(\alpha_{1}, \alpha_{2}, \ldots\right):=\left(\beta_{1}, \beta_{2}, \ldots\right) \quad \forall\left(\alpha_{1}, \alpha_{2}, \ldots\right) \in \ell^{\infty}
$$

where one has

$$
\beta_{k}= \begin{cases}\alpha_{j} & \text { if } k=k_{j}, j \in \mathbb{N} \\ 0 & \text { otherwise }\end{cases}
$$


Taking the above sequence $\left\{x_{j}^{*}\right\}$, we denote $z_{j}^{*}:=\left.x_{j}^{*}\right|_{z}$ and form a linear bounded operator $B: Z \rightarrow c_{0}$ by

$$
B(z):=\left(\left\langle z_{1}^{*}, z\right\rangle,\left\langle z_{2}^{*}, z\right\rangle, \ldots\right) \in c_{0} \quad \forall z \in Z
$$

Then the operator $(B \circ A): \ell^{\infty} \rightarrow c_{0}$ is bounded and its restriction $(B \circ A) \mid c_{0}$ is the identity operator on $c_{0}$. Therefore, $(B \circ A)$ is a projection of $\ell^{\infty}$ to $c_{0}$, which means that $c_{0}$ is complemented in $\ell^{\infty}$. It is well known that the latter is not true, and hence we get a contradiction. This proves that $c_{0}$ is not $w^{*}$-extensible in $\ell^{\infty}$.

We show that linear operators with $w^{*}$-extensible ranges enjoy a certain stability property, which is crucial for the subsequent applications in this paper.

Proposition 3.7. Let $A: X \rightarrow Y$ be a linear continuous operator between Banach spaces. Assume that $A(X)$ is closed and $w^{*}$-extensible in $Y$ and take $x_{k}^{*} \in A^{*}\left(Y^{*}\right)$ with $x_{k}^{*} \stackrel{w^{*}}{\longrightarrow} x^{*}$. Then $\left(A^{*}\right)^{-1}\left(x^{*}\right) \neq \varnothing$, and for every $y^{*} \in\left(A^{*}\right)^{-1}\left(x^{*}\right)$, there is a sequence $y_{k}^{*} \in\left(A^{*}\right)^{-1}\left(x_{k}^{*}\right)$ that contains a subsequence weak* convergent to $y^{*}$.

Proof. It is well known that the range $A^{*}\left(Y^{*}\right)$ of the adjoint operator to $A$ is weak* closed in $X^{*}$ if $L:=A(X)$ is closed in $Y$. Thus $x^{*} \in A^{*}\left(Y^{*}\right)$, that is, $\left(A^{*}\right)^{-1}\left(x^{*}\right) \neq \varnothing$. Take any $y^{*} \in\left(A^{*}\right)^{-1}\left(x^{*}\right)$, arbitrarily choose $\hat{y}_{k}^{*} \in\left(A^{*}\right)^{-1}\left(x_{k}^{*}\right)$, and let $v_{k}^{*}:=\left.\hat{y}_{k}^{*}\right|_{L}$. Then $\left.v_{k}^{*} \stackrel{w^{*}}{\longrightarrow} y^{*}\right|_{L}$ in $L^{*}$. Since the space $L$ is closed and $w^{*}$-extensible in $Y$, we find an extension $\tilde{y}_{k}^{*}$ of $v_{k}^{*}-\left.y^{*}\right|_{L}$ for each $k \in \mathbb{N}$ such that $\left\{\tilde{y}_{k}^{*}\right\}$ contains a subsequence that weak* converges to zero. Now letting $y_{k}^{*}:=y^{*}+\tilde{y}_{k}^{*}$, we check that $A^{*}\left(y_{k}^{*}\right)=x_{k}^{*}$ and that $\left\{y_{k}^{*}\right\}$ contains a subsequence weak* convergent to $y^{*}$.

THEOREM 3.8. Let $f: X \rightarrow Y$ be a mapping between Banach spaces. Assume that $f$ is strictly differentiable at $\bar{x}$ and RMR around this point, and that the derivative image $\nabla f(\bar{x})(X)$ is $w^{*}$-extensible in $Y$. Then for any $\Omega \subset Y$ with $f(\bar{x}) \in \Omega$,

$$
\begin{gathered}
N\left(\bar{x} ; f^{-1}(\Omega)\right)=\nabla f(\bar{x})^{*} N(f(\bar{x}) ; \Omega \cap f(X)), \\
\left(\nabla f(\bar{x})^{*}\right)^{-1} N\left(\bar{x} ; f^{-1}(\Omega)\right)=N(f(\bar{x}) ; \Omega \cap f(X)) .
\end{gathered}
$$

Moreover, $f^{-1}(\Omega)$ is normally regular at $\bar{x}$ if and only if $\Omega \cap f(X)$ has this property at $f(\bar{x})$.

Proof. Let $\bar{y}:=f(\bar{x})$ and pick any $y^{*} \in N(\bar{y} ; \Omega \cap f(X))$. Then using the definition of basic normals and the RMR property of $f$ around $\bar{x}$, we find sequences $\varepsilon_{k} \downarrow 0, x_{k} \rightarrow \bar{x}$, and $y_{k}^{*} \stackrel{w^{*}}{\longrightarrow} y^{*}$ such that

$$
x_{k} \in f^{-1}(\Omega), \quad y_{k}^{*} \in \hat{N}_{\varepsilon_{1 k}}\left(f\left(x_{k}\right) ; \Omega \cap f(X)\right), \quad \forall k \in \mathbb{N} .
$$

Applying inclusion (3.8) in Theorem 3.1 with $A:=\nabla f(\bar{x})$, we get

$$
\nabla f(\bar{x})^{*} y_{k}^{*} \in \hat{N}_{\varepsilon_{1 k}}\left(x_{k} ; f^{-1}(\Omega)\right), \quad \varepsilon_{1 k}:=\mu_{1} \varepsilon_{k}+\left\|y_{k}^{*}\right\| r_{f}\left(\bar{x} ;\left\|x_{k}-\bar{x}\right\|\right),
$$


where $r_{f}\left(\bar{x} ;\left\|x_{k}-\bar{x}\right\|\right) \rightarrow 0$ as $k \rightarrow \infty$ due to the strict differentiability of $f$ at $\bar{x}$. Since the sequence $\left\{y_{k}^{*}\right\}$ is uniformly bounded in $Y^{*}$ by the unform boundedness principle, we have $\varepsilon_{1 k} \downarrow 0$ as $k \rightarrow \infty$. Thus $\nabla f(\bar{x})^{*} y^{*} \in N\left(\bar{x} ; f^{-1}(\Omega)\right)$, which justifies the inclusion

$$
\nabla f(\bar{x})^{*} N(f(\bar{x}) ; \Omega \cap f(X)) \subset N\left(\bar{x} ; f^{-1}(\Omega)\right)
$$

and hence the one in

$$
\left(\nabla f(\bar{x})^{*}\right)^{-1} N\left(\bar{x} ; f^{-1}(\Omega)\right) \supset N(f(\bar{x}) ; \Omega \cap f(X))
$$

without the $w^{*}$-extensibility assumption on $\nabla f(\bar{x})(X)$. We prove that the opposite inclusion holds in (3.32) and hence in (3.31) under the latter assumption.

Picking $x^{*} \in N\left(\bar{x} ; f^{-1}(\Omega)\right)$, we find sequences $\varepsilon_{k} \downarrow 0, x_{k} \in f^{-1}(\Omega)$, and $x_{k}^{*} \in \widehat{N}_{\varepsilon_{k}}\left(x_{k}\right.$; $\left.f^{-1}(\Omega)\right)$ such that $x_{k} \rightarrow \bar{x}$ and $x_{k}^{*} \stackrel{w^{*}}{\longrightarrow} x^{*}$. By Theorem 3.1, there are $\varepsilon_{1 k} \downarrow 0$ and $\hat{x}_{k}^{*} \in$ $x_{k}^{*}+\varepsilon_{1 k} \mathbb{B}^{*}$ such that $\hat{x}_{k}^{*} \in \nabla f(\bar{x})^{*}\left(Y^{*}\right)$. Since $\hat{x}_{k}^{*} \stackrel{w^{*}}{\longrightarrow} x^{*}$, we conclude from Proposition 3.7 that $x^{*} \in \nabla f(\bar{x})^{*}\left(Y^{*}\right)$ and that for each $y^{*} \in\left(\nabla f(\bar{x})^{*}\right)^{-1}\left(x^{*}\right)$, there is a sequence $\left\{y_{k}^{*}\right\}$ with $y_{k}^{*} \in\left(\nabla f(\bar{x})^{*}\right)^{-1}\left(\hat{x}_{k}^{*}\right)$ that contains a subsequence $w^{*}$-convergent to $y^{*}$. Using again Theorem 3.1, we find $\varepsilon_{2 k} \downarrow 0$ such that $y_{k}^{*} \in \widehat{N}_{\varepsilon_{2 k}}\left(f\left(x_{k}\right) ; \Omega \cap f(X)\right)$. Thus $y^{*} \in N(f(\bar{x}) ; \Omega \cap f(X))$, which justifies the opposite inclusions in (3.32) and (3.31). The regularity statement follows directly from the normal cone formulas obtained in the theorem and Corollary 3.3.

COROLlary 3.9. Let $f: X \rightarrow Y$ be a mapping between Banach spaces that is strictly differentiable at $\bar{x}$ and such that $\nabla f(\bar{x})$ is surjective. Then

$$
N\left(\bar{x} ; f^{-1}(\Omega)\right)=\nabla f(\bar{x})^{*} N(f(\bar{x}) ; \Omega)
$$

for any $\Omega \subset Y$ with $f(\bar{x}) \in Y$.

Proof. Follows directly from Theorem 3.8 and the Lyusternik-Graves theorem.

The next theorem gives chain rules for the above coderivatives (3.3) as $\varepsilon=0$, (3.4), and (3.5) of mappings between Banach spaces. Given two set-valued mappings $F: X \rightrightarrows Y$ and $G: Y \rightrightarrows Z$, the restriction $\left.G\right|_{F}: Y \rightrightarrows Z$ of $G$ on $F$ is defined by

$$
\left.G\right|_{F}(y):= \begin{cases}G(y) & \text { if } y \in F(X), \\ \varnothing & \text { otherwise. }\end{cases}
$$

THEOREM 3.10. Let $f: X \rightarrow Y$ and $G: Y \rightrightarrows Z$ be mappings between Banach spaces, and let $\bar{z} \in G(f(\bar{x}))$. Assume that $f$ is strictly differentiable at $\bar{x}$ and RMR around this point. Then

$$
\begin{gathered}
\hat{D}^{*}(G \circ f)(\bar{x}, \bar{z})=\left.\nabla f(\bar{x})^{*} \circ \hat{D}^{*} G\right|_{f}(f(\bar{x}), \bar{z}), \\
\left(\nabla f(\bar{x})^{*}\right)^{-1} \circ \hat{D}^{*}(G \circ f)(\bar{x}, \bar{z})=\left.\hat{D}^{*} G\right|_{f}(f(\bar{x}), \bar{z}) .
\end{gathered}
$$


If, in addition, $\nabla f(\bar{x})(X)$ is $w^{*}$-extensible in $Y$, then

$$
\begin{gathered}
D^{*}(G \circ f)(\bar{x}, \bar{z})=\left.\nabla f(\bar{x})^{*} \circ D^{*} G\right|_{f}(f(\bar{x}), \bar{z}), \\
\left(\nabla f(\bar{x})^{*}\right)^{-1} \circ D^{*}(G \circ f)(\bar{x}, \bar{z})=\left.D^{*} G\right|_{f}(f(\bar{x}), \bar{z}),
\end{gathered}
$$

where $D^{*}$ stands for either $D_{N}^{*}$ or $D_{M}^{*}$. Moreover, the composition $G \circ f$ is $N$-regular (resp., $M$-regular) at $(\bar{x}, \bar{z})$ if and only if $\left.G\right|_{f}$ has the corresponding property at $(f(\bar{x}), \bar{z})$ under all the assumptions made.

Proof. Taking the identity operator $I$ on $Z$, we define $(f \oplus I): X \times Z \rightarrow Y \times Z$ by $(f \oplus I)(x, z):=(f(x), z)$ for any $x \in X$ and $z \in Z$. Then $\operatorname{gph}(G \circ f)=(f \oplus I)^{-1}(\operatorname{gph} G)$, and hence the chain rules of the theorem follow from Corollary 3.3 and Theorem 3.8. The regularity statement is clearly implied by the coderivative calculus rules.

If $\nabla f(\bar{x})$ is surjective, then Theorem 3.10 gives the chain rule

$$
D^{*}(G \circ f)(\bar{x}, \bar{z})=\nabla f(\bar{x})^{*} \circ D^{*} G(f(\bar{x}), \bar{z})
$$

for all the coderivatives under consideration with no other assumptions. Theorem 3.10 easily implies the subdifferential chain rules for the corresponding constructions defined in (3.6). In what follows, $\delta_{\Omega}(\cdot)$ stands for the indicator function of a set $\Omega$, that is, $\delta(x)=0$ if $x \in \Omega$ and $\delta(x ; \Omega)=\infty$ otherwise.

COROLLARY 3.11. Let $f: X \rightarrow Y$ be a mapping between Banach spaces that is strictly differentiable at $\bar{x}$, and let $\varphi: Y \rightarrow \overline{\mathbb{R}}$ be finite at $f(\bar{x})$. Assume that $f$ is RMR around $\bar{x}$. Then the following equalities hold:

$$
\begin{gathered}
\hat{\partial}(\varphi \circ f)(\bar{x})=\nabla f(\bar{x})^{*} \hat{\partial}\left(\varphi+\delta_{f(X)}\right)(f(\bar{x})), \\
\left(\nabla f(\bar{x})^{*}\right)^{-1} \hat{\partial}(\varphi \circ f)(\bar{x})=\hat{\partial}\left(\varphi+\delta_{f(X)}\right)(f(\bar{x})) .
\end{gathered}
$$

If, in addition, $\nabla f(\bar{x})(X)$ is $w^{*}$-extensible in $Y$, then

$$
\begin{gathered}
\partial(\varphi \circ f)(\bar{x})=\nabla f(\bar{x})^{*} \partial\left(\varphi+\delta_{f(X)}\right)(f(\bar{x})), \\
\left(\nabla f(\bar{x})^{*}\right)^{-1} \partial(\varphi \circ f)(\bar{x})=\partial\left(\varphi+\delta_{f(X)}\right)(f(\bar{x})) .
\end{gathered}
$$

Hence $\varphi \circ f$ is lower regular at $\bar{x}$ if and only if $\varphi+\delta_{f(X)}$ has this property at $f(\bar{x})$.

Proof. Follows from Theorem 3.10 with $G:=E_{\varphi}$.

4. Second-order calculus. Let $\varphi: X \rightarrow \overline{\mathbb{R}}$ be finite at $\bar{x}$, and let $\bar{y} \in \partial \varphi(\bar{x})$ for the basic first-order subdifferential defined in (3.6). In this section, we develop some calculus results for the second-order constructions defined, as set-valued mappings from $X^{* *}$ to $X^{*}$, by

$$
\begin{aligned}
& \partial_{N}^{2} \varphi(\bar{x}, \bar{y})(u):=\left(D_{N}^{*} \partial \varphi\right)(\bar{x}, \bar{y})(u), \\
& \partial_{M}^{2} \varphi(\bar{x}, \bar{y})(u):=\left(D_{M}^{*} \partial \varphi\right)(\bar{x}, \bar{y})(u),
\end{aligned}
$$


in terms of the normal and mixed coderivatives (3.4) and (3.5). Constructions (4.1) are called, respectively, the normal and mixed second-order subdifferentials of $\varphi$ at $\bar{x}$ relative to $\bar{y}$. We refer the reader to $[21,22]$ and the works cited therein for the theory and applications of these constructions in finite-dimensional and infinite-dimensional (mostly Asplund) spaces. It is clear that $\partial_{N}^{2} \varphi(\bar{x}, \bar{y})=\partial_{M}^{2} \varphi(\bar{x}, \bar{y}):=\partial^{2} \varphi(\bar{x}, \bar{y})$ when $X$ is finite dimensional, and that, in general, one has

$$
\partial_{N}^{2} \varphi(\bar{x}, \bar{y})(u)=\partial_{M}^{2} \varphi(\bar{x}, \bar{y})(u)=\left\{\nabla^{2} \varphi(\bar{x})^{*} u\right\}, \quad u \in X^{* *},
$$

for functions twice continuously differentiable around $\bar{x}$.

Our goal is to obtain chain rules for both second-order subdifferentials of (4.1) of compositions $\varphi \circ g$ involving smooth mappings $g: X \rightarrow Z$ and extended real-valued functions $\varphi: Z \rightarrow \overline{\mathbb{R}}$ in Banach space settings. To proceed, we first establish the following theorem that gives chain rules for coderivatives of special compositions whose structure as well as imposed assumptions are suitable for applications to second-order subdifferentials.

TheOREM 4.1. Let $G: X \rightrightarrows Y$ and $f: X \times Y \rightarrow Z$ be mappings between general Banach spaces. Consider the composition

$$
(f \circ G)(x):=f(x, G(x))=\bigcup\{f(x, y) \mid y \in G(x)\},
$$

and, given $\bar{x}$ with $G(\bar{x}) \neq \varnothing$, assume that

(a) $f(x, \cdot)$ is a bounded linear operator from $Y$ into $Z$ for every $x$ around $\bar{x}$. Moreover, $f(\bar{x}, \cdot)$ is injective with the closed range in $Z$;

(b) the mapping $x \rightarrow f(x, \cdot)$ from $X$ to the operator space $\mathscr{B}(Y, Z)$ is strictly differentiable at $\bar{x}$.

Take any $\bar{y} \in G(\bar{x})$ and denote $\bar{z}:=f(\bar{x}, \bar{y})$. Then

$$
\begin{aligned}
& D_{M}^{*}(f \circ G)(\bar{x}, \bar{z})\left(z^{*}\right)=\nabla_{x} f(\bar{x}, \bar{y})^{*} z^{*}+D_{M}^{*} G(\bar{x}, \bar{y})\left(f(\bar{x}, \cdot)^{*} z^{*}\right), \\
& D_{N}^{*}(f \circ G)(\bar{x}, \bar{z})\left(z^{*}\right) \subset \nabla_{x} f(\bar{x}, \bar{y})^{*} z^{*}+D_{N}^{*} G(\bar{x}, \bar{y})\left(f(\bar{x}, \cdot)^{*} z^{*}\right),
\end{aligned}
$$

for all $z^{*} \in Z^{*}$. If, in addition, the range of $f(\bar{x}, \cdot)$ is $w^{*}$-extensible in $Z$, then inclusion (4.5) holds as equality.

Proof. Consider the mapping $h(x):=f(x, \cdot)$ from $X$ to $\mathscr{B}(Y, Z)$ and denote by $A: X \rightarrow \mathscr{B}(Y, Z)$ its strict derivative at $\bar{x}$. Let $\ell>0$ be a Lipschitz modulus of $h$ around $\bar{x}$. For any $y \in Y$, we define a linear operator $A_{y}: X \rightarrow Z$ by $A_{y}(x):=A(x) y$ and easily check that it is bounded. Moreover, the operator $y \rightarrow A_{y}$ from $Y$ to $\mathscr{B}(X, Z)$ is linear and bounded as well. By enlarging $\ell$, if necessary, we assume that the norm of this operator is less than $\ell$. Also, it is clear that $A_{y}=\nabla_{x} f(\bar{x}, y)$ for all $y \in Y$.

Our first step is to prove the inclusions " $\subset$ " in (4.4) and (4.5) simultaneously. Proceeding by definitions of these coderivatives, we start with $\varepsilon$-normals

$$
\left(x^{*},-z^{*}\right) \in \hat{N}_{\varepsilon}((\hat{x}, \hat{z}) ; \operatorname{gph}(f \circ G)),
$$


where $\hat{z}:=f(\hat{x}, \hat{y}),(\hat{x}, \hat{y}) \in \operatorname{gph} G$ with $\|\hat{x}-\bar{x}\|<\eta$ for some small $\eta>0$. Using the definition of $\varepsilon$-normals and involving the rate of strict differentiability $r_{h}(\bar{x} ; \eta)$ for the above mapping $h$ at $\bar{x}$ (see Section 2), we get

$$
\limsup _{(x, y) \stackrel{G}{\rightarrow}(\hat{x}, \hat{y})} \frac{\left\langle x^{*}-A_{\bar{y}}^{*} z^{*}, x-\bar{x}\right\rangle-\left\langle f(\bar{x}, \cdot)^{*} z^{*}, y-\hat{y}\right\rangle}{\|x-\hat{x}\|+\|y-\hat{y}\|} \leq \hat{\varepsilon},
$$

where $\hat{\varepsilon}:=c \varepsilon+c\left\|z^{*}\right\|\left(r_{h}(\bar{x} ; \eta)+\|\hat{x}-\bar{x}\|+\|\hat{y}-\bar{y}\|\right)$ with some constant $c>0$. Thus one has

$$
\left(x^{*}-A_{\bar{y}}^{*} z^{*},-f(\bar{x}, \cdot)^{*} z^{*}\right) \in \hat{N}_{\hat{\varepsilon}}((\hat{x}, \hat{y}) ; \operatorname{gph} G) .
$$

To justify the inclusions " $\subset$ " in (4.4) and (4.5) simultaneously, we take $x^{*} \in D^{*}(f \circ$ $G)(\bar{x}, \bar{z})\left(z^{*}\right)$ and find sequences $\varepsilon_{k} \downarrow 0, x_{k} \rightarrow \bar{x}, y_{k} \in G\left(x_{k}\right)$, and $\left(x_{k}^{*},-z_{k}^{*}\right) \in \hat{N}\left(\left(x_{k}, z_{k}\right)\right.$; $\operatorname{gph}(f \circ G))$ with $z_{k}:=f\left(x_{k}, y_{k}\right)$ satisfying $z_{k} \rightarrow \bar{z}$ and $x_{k}^{*} \stackrel{w^{*}}{\longrightarrow} x^{*}$ and such that $\| z_{k}^{*}-$ $z^{*} \| \rightarrow 0$ for $D^{*}=D_{M}^{*}$ and $z_{k}^{*} \stackrel{w^{*}}{\longrightarrow} z^{*}$ for $D^{*}=D_{N}^{*}$. Then we obtain the inclusions in (4.4) and (4.5) by passing to the limit in (4.8) provided that $y_{k} \rightarrow \bar{y}$. To prove the latter convergence, we observe that the open mapping theorem and the injectivity of $f(\bar{x}, \cdot)$ ensure the existence of a constant $\mu>0$ with

$$
\|f(\bar{x}, u)-f(\bar{x}, v)\| \geq \mu\|u-v\| \quad \text { whenever } u, v \in Y .
$$

Therefore, involving the above Lipschitz modulus $\ell$, one has

$$
\begin{aligned}
\left\|z_{k}-\bar{z}\right\|= & \|\left[f\left(\bar{x}, y_{k}\right)-f(\bar{x}, \bar{y})\right]+\left[f\left(x_{k}, y_{k}-\bar{y}\right)-f\left(\bar{x}, y_{k}-\bar{y}\right)\right] \\
& +\left[f\left(x_{k}, \bar{y}\right)-f(\bar{x}, \bar{y})\right] \| \\
\geq & \left\|y_{k}-\bar{y}\right\|\left(\mu-\ell\left\|x_{k}-\bar{x}\right\|\right)-\ell\left\|x_{k}-\bar{x}\right\| \cdot\|\bar{y}\|,
\end{aligned}
$$

which implies that $y_{k} \rightarrow \bar{y}$ as $k \rightarrow \infty$.

Next, we show that the opposite inclusions hold in (4.4) and (4.5) under the assumptions made; in fact, there are no additional assumptions in the case of mixed coderivatives (4.4). To proceed simultaneously in both cases, we take $(\hat{x}, \hat{y})$ as above and pick arbitrary $\left(x^{*}, z^{*}\right)$ satisfying

$$
\left(x^{*},-f(\bar{x}, \cdot)^{*} z^{*}\right) \in \hat{N}_{\varepsilon}((\hat{x}, \hat{y}) ; \mathrm{gph} G) .
$$

Thus, for any given $\gamma>0$, one has

$$
\theta:=\left\langle x^{*}, x-\hat{x}\right\rangle-\left\langle f(\bar{x}, \cdot)^{*} z^{*}, y-\hat{y}\right\rangle \leq(\varepsilon+\gamma)(\|x-\hat{x}\|+\|y-\hat{y}\|)
$$

whenever $(x, y) \in \operatorname{gph} G$ are sufficiently close to $(\hat{x}, \hat{y})$. We obtain a lower estimate for $\theta$ in (4.12) using the strict differentiability of the above mapping $h: X \rightarrow \mathscr{B}(Y, Z)$ at $\bar{x}$ 
with the rate $r_{h}(\bar{x} ; \eta)$ and elementary transformations. In this way, we get

$$
\begin{aligned}
\theta= & \left\langle x^{*}, x-\hat{x}\right\rangle-\left\langle z^{*}, f(\bar{x}, y)-f(\bar{x}, \hat{y})\right\rangle \\
= & \left\langle x^{*}+A_{\bar{y}}^{*} z^{*}, x-\hat{x}\right\rangle-\left\langle z^{*}, A_{\bar{y}}(x-\hat{x})\right\rangle-\left\langle z^{*}, f(\bar{x}, y)-f(\bar{x}, \hat{y})\right\rangle \\
\geq & \left\langle x^{*}+A_{\bar{y}}^{*} z^{*}, x-\hat{x}\right\rangle-\left\langle z^{*}, A_{y}(x-\hat{x})\right\rangle-\left\langle z^{*}, f(\hat{x}, y)-f(\hat{x}, \hat{y})\right\rangle \\
& -\ell\left\|z^{*}\right\| \cdot\|y-\bar{y}\| \cdot\|x-\hat{x}\|-\ell\left\|z^{*}\right\| \cdot\|\hat{x}-\bar{x}\| \cdot\|y-\hat{y}\| \\
\geq & \left\langle x^{*}+A_{\bar{y}}^{*} z^{*}, x-\hat{x}\right\rangle-\left\langle z^{*}, f(x, y)-f(\hat{x}, y)\right\rangle \\
& -r_{h}(\bar{x} ; \eta)\left\|z^{*}\right\| \cdot\|y\| \cdot\|x-\hat{x}\|-\left\langle z^{*}, f(\hat{x}, y)-f(\hat{x}, \hat{y})\right\rangle \\
& -\ell\left\|z^{*}\right\|(\|y-\bar{y}\| \cdot\|x-\hat{x}\|+\|\hat{x}-\bar{x}\| \cdot\|y-\hat{y}\|) \\
= & \left\langle x^{*}+A_{y}^{*} z^{*}, x-\hat{x}\right\rangle-\left\langle z^{*}, f(x, y)-f(\hat{x}, \hat{y})\right\rangle \\
& -r_{h}(\bar{x} ; \eta)\left\|z^{*}\right\| \cdot\|y\| \cdot\|x-\hat{x}\| \\
& -\ell\left\|z^{*}\right\|(\|y-\bar{y}\| \cdot\|x-\hat{x}\|+\|\hat{x}-\bar{x}\| \cdot\|y-\hat{y}\|) .
\end{aligned}
$$

Now we are going to give an upper estimate of the number on the right-hand side of (4.12). To proceed, we first observe that, by the open mapping theorem and the injectivity of $f(\bar{x}, \cdot)$, there is $\mu>0$ such that

$$
\mu\|y\| \leq\|f(\bar{x}, y)\| \quad \forall y \in Y
$$

Then taking any $T \in \mathscr{B}(Y, Z)$, we get

$$
\begin{aligned}
\|T y\| & =\|(f(\bar{x}, \cdot)-T) y-f(\bar{x}, y)\| \geq\|f(\bar{x}, y)\|-\|(f(\bar{x}, \cdot)-T) y\| \\
& \geq(\mu-\|f(\bar{x}, \cdot)-T\|) \cdot\|y\| .
\end{aligned}
$$

This implies the existence of a constant $\mu_{1}>0$ with the uniform estimate $\mu_{1}\|y\| \leq\|T y\|$ for all $y \in Y$ and all $T$ sufficiently close to $f(\bar{x}, \cdot)$. It gives therefore that

$$
\begin{aligned}
\|f(x, y)-f(\hat{x}, \hat{y})\| & =\|f(x, y)-f(\hat{x}, y)+f(\hat{x}, y-\hat{y})\| \\
& \geq\|f(\hat{x}, y-\hat{y})\|-\|f(x, y)-f(\hat{x}, y)\| \\
& \geq \mu_{1}\|y-\hat{y}\|-L\|x-\hat{x}\| \cdot\|y\|
\end{aligned}
$$

for $(x, y) \in \operatorname{gph} G$ close to $(\hat{x}, \hat{y})$ while $(\hat{x}, \hat{y})$ is close to $(\bar{x}, \bar{y})$. Thus we obtain the estimate

$$
\|y-\hat{y}\| \leq \mu_{2}(\|x-\hat{x}\|+\|f(x, y)-f(\hat{x}, \hat{y})\|)
$$

for all such $(x, y)$ and $(\hat{x}, \hat{y})$, with some constant $\mu_{2}>0$. Putting these estimates together, one has the inclusion

$$
\left(x^{*}+A_{\bar{y}}^{*} z^{*},-z^{*}\right) \in \hat{N}_{\hat{\varepsilon}}((\hat{x}, \hat{z}) ; \operatorname{gph}(f \circ G)),
$$

where $\hat{z}:=f(\hat{x}, \hat{y})$ and $\hat{\varepsilon}$ is defined as above with a different constant $c>0$. 
To prove the opposite inclusions in (4.4) and (4.5), we need to pass to the limit in $(4.18)$ as $(\hat{x}, \hat{y}) \rightarrow(\bar{x}, \bar{y})$ along some sequence. Pick arbitrary $\left(x^{*}, z^{*}\right)$ with $x^{*} \in$ $D^{*} G(\bar{x}, \bar{y})\left(f(\bar{x}, \cdot)^{*} z^{*}\right)$, where $D^{*}$ stands for either mixed or normal coderivative. Then there are sequences $\varepsilon_{k} \downarrow 0,\left(x_{k}, y_{k}\right) \rightarrow(\bar{x}, \bar{y})$ with $\left(x_{k}, y_{k}\right) \in \operatorname{gph} G$, and $x_{k}^{*} \in D_{\varepsilon_{k}}^{*} G\left(x_{k}\right.$, $\left.y_{k}\right)\left(y_{k}^{*}\right)$ such that $x_{k}^{*} \stackrel{w^{*}}{\longrightarrow} x^{*}$, and either $\left\|y_{k}^{*}-f(\bar{x}, \cdot)^{*} z^{*}\right\| \rightarrow 0$ when $D^{*}=D_{M}^{*}$ or $y_{k}^{*} \stackrel{w^{*}}{\longrightarrow} f(\bar{x}, \cdot)^{*} z^{*}$ when $D^{*}=D_{N}^{*}$. Note that $\hat{\varepsilon}_{k} \downarrow 0$ for the corresponding $\hat{\varepsilon}_{k}$ in (4.18). To complete the proof of the theorem, it is sufficient to show that there are $z_{k}^{*} \in Z^{*}$ such that $f(\bar{x}, \cdot)^{*} z_{k}^{*}=y_{k}^{*}$ for all $k \in \mathbb{N}$, and that either $\left\|z_{k}^{*}-z^{*}\right\| \rightarrow 0$ for $D^{*}=D_{M}^{*}$ or $z_{k}^{*} \stackrel{w^{*}}{\longrightarrow} z^{*}$ for $D^{*}=D_{N}^{*}$ along a subsequence. We consider the cases of mixed and normal coderivatives separately.

(i) Let $D^{*}=D_{M}^{*}$. Since $f(\bar{x}, \cdot)$ is injective with the closed range, it is easy to see that the adjoint operator $f(\bar{x}, \cdot)^{*}$ is surjective and hence metrically regular. This ensures the existence of $\mu>0$ and $\hat{z}_{k}^{*} \in\left(f(\bar{x}, \cdot)^{*}\right)^{-1}\left(y_{k}^{*}-f(\bar{x}, \cdot)^{*} z^{*}\right)$ satisfying

$$
\left\|\hat{z}_{k}^{*}\right\| \leq \mu\left\|y_{k}^{*}-f(\bar{x}, \cdot)^{*} z^{*}\right\| .
$$

Putting $z_{k}^{*}:=\hat{z}_{k}^{*}+z^{*}$, we get $f(\bar{x}, \cdot)^{*} z_{k}^{*}=y_{k}^{*}$ and $\left\|z_{k}^{*}-z^{*}\right\| \rightarrow 0$ as $k \rightarrow \infty$.

(ii) Let $D^{*}=D_{N}^{*}$. In this case, the subspace $f(\bar{x}, Y)$ is assumed to be $w^{*}$-extensible in $Z$. Then the existence of the desired sequence $\left\{z_{k}^{*}\right\}$ follows from Proposition 3.7.

Now we are ready to derive second-order subdifferential chain rules for both constructions in (4.1) in general Banach space settings.

THEOREM 4.2. Let $X$ and $Z$ be Banach spaces, and let $\bar{y} \in \partial(\varphi \circ g)(\bar{x})$ with $g: X \rightarrow Z$ and $\varphi: Z \rightarrow \mathbb{R}$. Assume that $g$ is continuously differentiable around $\bar{x}$ with the surjective derivative $\nabla g(\bar{x}): X \rightarrow Z$ and that the mapping $\nabla g: X \rightarrow \mathscr{B}(X, Z)$ is strictly differentiable at $\bar{x}$. Let $\bar{v} \in Z^{*}$ be a unique functional satisfying the relations

$$
\bar{y}=\nabla g(\bar{x})^{*} \bar{v}, \quad \bar{v} \in \partial \varphi(\bar{z}), \quad \text { with } \bar{z}:=g(\bar{x}) .
$$

Then for all $u \in X^{* *}$,

$$
\begin{aligned}
& \partial_{M}^{2}(\varphi \circ g)(\bar{x}, \bar{y})(u)=\nabla^{2}\langle\bar{v}, g\rangle(\bar{x})^{*} u+\nabla g(\bar{x})^{*} \partial_{M}^{2} \varphi(\bar{z}, \bar{v})\left(\nabla g(\bar{x})^{* *} u\right), \\
& \partial_{N}^{2}(\varphi \circ g)(\bar{x}, \bar{y})(u) \subset \nabla^{2}\langle\bar{v}, g\rangle(\bar{x})^{*} u+\nabla g(\bar{x})^{*} \partial_{N}^{2} \varphi(\bar{z}, \bar{v})\left(\nabla g(\bar{x})^{* *} u\right) .
\end{aligned}
$$

Moreover, the latter inclusion becomes an equality if the range of $\nabla g(\bar{x})^{*}$ is $w^{*}$ extensible in $X^{*}$. This is true under one of the following conditions:

(a) the range of $\nabla g(\bar{x})^{*}$ is complemented in $X^{*}$, which holds, in particular, when the kernel of $\nabla g(\bar{x})$ is complemented in $X$;

(b) the closed unit ball of $X^{* *}$ is weak* sequentially compact, which holds, in particular, when either $X$ is reflexive or $X^{*}$ is separable. 
Proof. Using the first-order subdifferential sum rule from Corollary 3.11, we have

$$
\partial(\varphi \circ g)(x)=\nabla g(x)^{*} \partial \varphi(g(x)):=(f \circ G)(x)
$$

for all $x$ around $\bar{x}$, where the mappings $f: X \times Z^{*} \rightarrow X^{*}$ and $G: X \rightrightarrows Z^{*}$ are defined by

$$
f(x, v):=\nabla g(x)^{*} v, \quad G(x):=\partial \varphi(g(x)) .
$$

Thus we represent $\partial(\varphi \circ g)$ as composition (4.3) and apply Theorem 4.1 to this composition. We check that its assumptions hold under the assumptions made in the theorem. Actually the only assumption we need to check is the injectivity of $\nabla g(\bar{x})^{*}$ : $Z^{*} \rightarrow X^{*}$, which is implied by the surjectivity of $\nabla g(\bar{x})$. The sufficient conditions for the $w^{*}$-extensibility property of $\nabla g(\bar{x})^{*}$ in $X^{*}$ listed in the theorem follow from Proposition 3.5.

5. Applications to sequential normal compactness. The primary goal of this section is to develop applications of the results obtained in Sections 2 and 4 to the so-called normal compactness properties of sets, functions, and set-valued mappings that play a principal role in infinite-dimensional variational analysis and its applications; see, in particular, $[2,3,4,10,11,14,16,20,24,25,26,27,28,29,30,31]$ and the references therein. Being automatic in finite dimensions, such properties are crucial for performing limiting procedures that involve weak* convergence in dual spaces and allow one to arrive at nontrivial point-based conclusions in generalized differential calculus, necessary optimality conditions, criteria for stability and metric regularity properties in general nonsmooth settings, and so forth. The reader may find more details and discussions in the works cited above.

In this section, we focus on SNC properties in the vein of those formulated in [24]. Developing a geometric approach and aiming to cover set-valued mappings and extended real-valued functions via sets, we primarily consider the following modified versions of partial SNC properties for subsets of Banach spaces endowed with a product structure; see $[26,27]$ for more discussions.

Given $\Omega \subset X_{1} \times X_{2}$, we say that $\Omega$ is partially sequentially normally compact (PSNC) at $\bar{x} \in \Omega$ with respect to $X_{1}$ if for any sequences

$$
\varepsilon_{k} \downarrow 0, \quad x_{k} \stackrel{\Omega}{\longrightarrow} \bar{x}, \quad\left(x_{1 k}^{*}, x_{2 k}^{*}\right) \in \widehat{N}_{\varepsilon_{k}}\left(x_{k} ; \Omega\right), \quad k \in \mathbb{N},
$$

with $x_{1 k}^{*} \stackrel{w^{*}}{\longrightarrow} 0$ and $\left\|x_{2 k}^{*}\right\| \rightarrow 0$, one has $\left\|x_{1 k}^{*}\right\| \rightarrow 0$ as $k \rightarrow \infty$. The set $\Omega$ is strongly PSNC at $\bar{x}$ with respect to $X_{1}$ if for any sequences $\left(\varepsilon_{k}, x_{k}, x_{1 k}^{*}, x_{2 k}^{*}\right)$ satisfying (5.1), one has

$$
\left[\left(x_{1 k}^{*}, x_{2 k}^{*}\right) \stackrel{w^{*}}{\longrightarrow}(0,0)\right] \Longrightarrow\left\|x_{1 k}^{*}\right\| \longrightarrow 0 \quad \text { as } k \longrightarrow \infty .
$$

When $X_{2}=\{0\}$, that is, there is no product structure on $X_{1}=X$, both definitions above reduce to the SNC property of $\Omega \subset X$ at $\bar{x}$. The latter property is closely related to (being generally weaker than) the compactly epi-Lipschitzian (CEL) property introduced by Borwein and Strójwas [3] as a generalization of the epi-Lipschitzian property by 
Rockafellar [32]. We refer the reader to [10] for characterizations of the CEL property in terms of topological (i.e., net) convergences of generalized normals and to [8] for a detailed comparison between the CEL and SNC properties in Banach and Asplund spaces.

SNC properties of set-valued mappings and extended real-valued functions are naturally introduced through the corresponding properties of their graphical and epigraphical sets. Given $F: X \rightrightarrows Y$, we say that it is $S N C$ at $(\bar{x}, \bar{y}) \in \operatorname{gph} F$ if its graph is SNC at $(\bar{x}, \bar{y})$ in the sense of the above definition. The mapping $F$ is PSNC at $(\bar{x}, \bar{y})$ if its graph is PSNC at this point with respect to $X$. It follows from [23, Theorem 5.8] that a set-valued mapping $F$ between general Banach spaces is PSNC at $(\bar{x}, \bar{y})$ if is satisfies the Aubin "pseudo-Lipschitzian" property [1] around this point. Finally, an extended real-valued function $\varphi: X \rightarrow \overline{\mathbb{R}}$ finite at $\bar{x}$ is sequentially normally epi-compact (SNEC) at this point if its epigraph is SNC at $(\bar{x}, \varphi(\bar{x}))$. It happens, in particular, when $\varphi$ is directionally Lipschitzian at $\bar{x}$ in the sense of Rockafellar [32].

To apply the SNC properties to specific problems, one needs to develop an SNC calculus, that is, to derive efficient conditions ensuring the preservation of these properties under various operations on sets and mappings. We have recently developed in [29] a fairly rich SNC calculus in the framework of Asplund spaces. We now present some results on the preservation of the SNC properties under inverse images of sets in general Banach spaces. Recall that, given two mappings $f_{i}: X_{i} \rightarrow Y_{i}, i=1,2$, between Banach spaces, $f_{1} \oplus f_{2}$ denotes a mapping from $X_{1} \oplus X_{2}$ to $Y_{1} \oplus Y_{2}$ with $\left(f_{1} \oplus f_{2}\right)\left(x_{1}, x_{2}\right):=$ $\left(f_{1}\left(x_{1}\right), f_{2}\left(x_{2}\right)\right)$ for all $x_{1} \in X_{1}, x_{2} \in X_{2}$.

THEOREM 5.1. Let $f_{i}: X_{i} \rightarrow Y_{i}, i=1,2$, be mappings between Banach spaces, let $\Omega$ be a subset of $Y_{1} \times Y_{2}$ with $\left(f_{1}\left(\bar{x}_{1}\right), f_{2}\left(\bar{x}_{2}\right)\right) \in \Omega$ for some $\left(\bar{x}_{1}, \bar{x}_{2}\right) \in X_{1} \times X_{2}$, and let $f:=f_{1} \oplus f_{2}$. Assume that each $f_{i}$ is strictly differentiable at $\bar{x}_{i}$ and $R M R$ around this point. Then the following hold.

(i) If $\Omega \cap f\left(X_{1} \times X_{2}\right)$ is PSNC at $f\left(\bar{x}_{1}, \bar{x}_{2}\right)$ with respect to $Y_{1}$, then $f^{-1}(\Omega)$ is PSNC at $\left(\bar{x}_{1}, \bar{x}_{2}\right)$ with respect to $X_{1}$. If, in addition, $\nabla f_{2}\left(\bar{x}_{2}\right)\left(X_{2}\right)$ is $w^{*}$-extensible in $Y_{2}$, then the strong PSNC property of $\Omega \cap f\left(X_{1} \times X_{2}\right)$ at $f\left(\bar{x}_{1}, \bar{x}_{2}\right)$ with respect to $Y_{1}$ implies that $f^{-1}(\Omega)$ is strongly PSNC at $\left(\bar{x}_{1}, \bar{x}_{2}\right)$ with respect to $X_{1}$.

(ii) Assume that $\nabla f_{1}\left(\bar{x}_{1}\right)\left(X_{1}\right)$ is of finite codimension in $Y_{1}$. Then conversely the PSNC (resp., strong PSNC) property of $f^{-1}(\Omega)$ at $\left(\bar{x}_{1}, \bar{x}_{2}\right)$ with respect to $X_{1}$ implies that $\Omega \cap$ $f\left(X_{1} \times X_{2}\right)$ is PSNC (resp., strongly PSNC) at $f\left(\bar{x}_{1}, \bar{x}_{2}\right)$ with respect to $Y_{1}$.

Proof. First we prove (i) assuming that $\Omega \cap f\left(X_{1} \times X_{2}\right)$ is PSNC at $\left(\bar{y}_{1}, \bar{y}_{2}\right):=f\left(\bar{x}_{1}\right.$, $\left.\bar{x}_{2}\right)$ with respect to $Y_{1}$. Since $f_{1}$ is RMR around $\bar{x}_{1}$, the linear subspace $L:=\nabla f_{1}\left(\bar{x}_{1}\right)\left(X_{1}\right)$ is closed in $Y_{1}$ by Theorem 2.2. Let $L^{\perp} \subset Y_{1}^{*}$ be the annihilator of $L$, that is, the set of all linear continuous functionals on $Y_{1}$ that vanish on $L$. It is well known that $L^{\perp}$ is isometric to the dual space of the quotient space $Y_{1} / L$. We show that $L$ is of finite codimension. Assume the contrary, that is, $\operatorname{dim}\left(Y_{1} / L\right)=\infty$. Then by the fundamental Josefson-Nissenzweig theorem (see., e.g., [5]), there is a sequence $\left\{y_{k}^{*}\right\} \subset\left(Y_{1} / L\right)^{*}$ that $w^{*}$-converges to zero with $\left\|y_{k}^{*}\right\|=1$ for all $k \in \mathbb{N}$. Using the above isomorphism, we can treat each $y_{k}^{*}$ as an element in $L^{\perp}$. Thus $y_{k}^{*} \stackrel{w^{*}}{\longrightarrow} 0$ in $Y_{1}^{*}$ with $\left\|y_{k}^{*}\right\|=1$. Employing the implication $(\mathrm{a}) \Rightarrow(\mathrm{b})$ in Theorem 2.10 and the definition of Fréchet normals, we check 
that

$$
\left(y_{k}^{*}, 0\right) \in \hat{N}\left(\left(\bar{y}_{1}, \bar{y}_{2}\right) ; \Omega \cap f\left(X_{1} \times X_{2}\right)\right) .
$$

Then the assumed PSNC property of $\Omega \cap f\left(X_{1} \times X_{2}\right)$ at $\left(\bar{y}_{1}, \bar{y}_{2}\right)$ with respect to $Y_{1}$ gives $\left\|y_{k}^{*}\right\| \rightarrow 0$, which is a contradiction. Thus $L$ is finite codimensional and hence $w^{*}$ extensible in $Y_{1}$ by Proposition 3.5(a). Now take arbitrary sequences

$$
\begin{gathered}
\varepsilon_{k} \downarrow 0, \quad\left(x_{1 k}, x_{2 k}\right) \in f^{-1}(\Omega), \\
\left(x_{1 k}^{*}, x_{2 k}^{*}\right) \in \hat{N}_{\varepsilon_{k}}\left(\left(x_{1 k}, x_{2 k}\right) ; f^{-1}(\Omega)\right)
\end{gathered}
$$

with $\left(x_{1 k}, x_{2 k}\right) \rightarrow\left(\bar{x}_{1}, \bar{x}_{2}\right), x_{1 k}^{*} \stackrel{w^{*}}{\longrightarrow} 0$, and $\left\|x_{2 k}^{*}\right\| \rightarrow 0$ as $k \rightarrow \infty$. By Theorem 3.1, there exist sequences $\tilde{x}_{i k}^{*} \stackrel{\|\cdot\|}{\longrightarrow} 0$ with $x_{i k}^{*}+\tilde{x}_{i k}^{*} \in \nabla f_{i}\left(\bar{x}_{i}\right)^{*}\left(Y_{i}^{*}\right)$ for $i=1$,2. Using the $w^{*}$ extensibility property mentioned above and applying Proposition 3.7, we find a sequence $\left\{y_{1 k}^{*}\right\}$ that contains a subsequence weak* convergent to $0 \in Y_{1}^{*}$ and such that $\nabla f_{1}\left(\bar{x}_{1}\right)^{*} y_{1 k}^{*}=x_{1 k}^{*}+\tilde{x}_{1 k}^{*}$. To justify the desired PSNC property, it is sufficient to show that $\left\{x_{1 k}^{*}\right\}$ contains a subsequence norm-convergent to zero; so we do not restrict the generality assuming that $y_{1 k}^{*} \stackrel{w^{*}}{\longrightarrow} 0$ as $k \rightarrow \infty$. On the other hand, take an arbitrary sequence of

$$
\tilde{y}_{2 k}^{*} \in\left(\nabla f_{2}\left(\bar{x}_{2}\right)^{*}\right)^{-1}\left(x_{2 k}^{*}+\tilde{x}_{2 k}^{*}\right), \quad k \in \mathbb{N},
$$

and observe that $\nabla f_{2}\left(\bar{x}_{2}\right)\left(X_{2}\right)$ is closed in $Y_{2}$ by Theorem 2.2, since $f_{2}$ is RMR around $\bar{x}_{2}$. Applying the classical open mapping theorem to the linear operator $\nabla f_{2}\left(\bar{x}_{2}\right): X_{2} \rightarrow$ $\nabla f_{2}\left(\bar{x}_{2}\right)\left(X_{2}\right)$, we find $\mu>0$ such that for any $y \in \nabla f_{2}\left(\bar{x}_{2}\right)\left(X_{2}\right)$, there is $x_{y} \in X_{2}$ with $\left\|x_{y}\right\| \leq \mu\|y\|$. Denoting $\hat{y}_{2 k}^{*}:=\left.\tilde{y}_{2 k}^{*}\right|_{\nabla f_{2}\left(\bar{x}_{2}\right)\left(X_{2}\right)}$, we have $\hat{y}_{2 k}^{*} \in \nabla f_{2}\left(\bar{x}_{2}\right)\left(X_{2}\right)^{*}$ and

$$
\begin{aligned}
\left|\left\langle\hat{y}_{2 k}^{*}, y\right\rangle\right| & =\left|\left\langle\tilde{y}_{2 k}^{*}, \nabla f_{2}\left(\bar{x}_{2}\right)\left(x_{y}\right)\right\rangle\right|=\left|\left\langle\nabla f_{2}\left(\bar{x}_{2}\right)^{*} \tilde{y}_{2 k}^{*}, x_{y}\right\rangle\right| \\
& =\left|\left\langle x_{2 k}^{*}+\tilde{x}_{2 k}^{*}, x_{y}\right\rangle\right| \leq\left\|x_{2 k}^{*}+\tilde{x}_{2 k}^{*}\right\| \cdot\left\|x_{y}\right\| \leq \mu\left\|x_{2 k}^{*}+\tilde{x}_{2 k}^{*}\right\| \cdot\|y\|
\end{aligned}
$$

for all $y \in \nabla f_{2}\left(\bar{x}_{2}\right)\left(X_{2}\right)$; thus $\left\|\hat{y}_{2 k}^{*}\right\| \leq \mu\left\|x_{2 k}^{*}+\tilde{x}_{2 k}^{*}\right\|$. The Hahn-Banach theorem allows us to extend each $\hat{y}_{2 k}^{*}$ to $y_{2 k}^{*} \in Y_{2}^{*}$ preserving the norm. By construction, one has $\left\|y_{2 k}^{*}\right\| \rightarrow 0$ as $k \rightarrow \infty$ and $\nabla f_{2}\left(\bar{x}_{2}\right)^{*} y_{2 k}^{*}=x_{2 k}^{*}+\tilde{x}_{2 k}^{*}$. Employing now Theorem 3.1, we find $\tilde{\varepsilon}_{k} \downarrow 0$ such that

$$
\left(y_{1 k}^{*}, y_{2 k}^{*}\right) \in \hat{N}_{\tilde{\varepsilon}_{k}}\left(\left(f_{1}\left(x_{1 k}\right), f_{2}\left(x_{2 k}\right)\right) ; \Omega \cap f\left(X_{1} \times X_{2}\right)\right) .
$$

It follows from the assumed PSNC property of $\Omega \cap f\left(X_{1} \times X_{2}\right)$ at $\left(\bar{y}_{1}, \bar{y}_{2}\right)$ with respect to $Y_{1}$ that $\left\|y_{1 k}^{*}\right\| \rightarrow 0$, which implies $\left\|x_{1 k}^{*}+\tilde{x}_{1 k}^{*}\right\| \rightarrow 0$ and hence $\left\|x_{1 k}^{*}\right\| \rightarrow 0$ as $k \rightarrow \infty$. This justifies the PSNC property of $f^{-1}(\Omega)$ at $\left(\bar{x}_{1}, \bar{x}_{2}\right)$ with respect to $X_{1}$.

The proof of the strong PSNC assertion in (i) can be conducted by similar arguments. The only difference is that now we use the $w^{*}$-extensibility property of $\nabla f_{2}\left(\bar{x}_{2}\right)\left(X_{2}\right)$ to build a sequence $\left\{y_{2 k}^{*}\right\}$ with $y_{2 k}^{*} \stackrel{w^{*}}{\longrightarrow} 0$ as $k \rightarrow \infty$. 
Finally, we prove part (ii) of the theorem focusing on the case of the PSNC property. Consider arbitrary sequences

$$
\begin{gathered}
\varepsilon_{k} \downarrow 0, \quad\left(y_{1 k}, y_{2 k}\right) \in \Omega \cap f\left(X_{1} \times X_{2}\right), \\
\left(y_{1 k}^{*}, y_{2 k}^{*}\right) \in \hat{N}_{\varepsilon_{k}}\left(\left(y_{1 k}, y_{2 k}\right) ; \Omega \cap f\left(X_{1} \times X_{2}\right)\right)
\end{gathered}
$$

with $\left(y_{1 k}, y_{2 k}\right) \rightarrow\left(\bar{y}_{1}, \bar{y}_{2}\right), y_{1 k}^{*} \stackrel{w^{*}}{\longrightarrow} 0$, and $\left\|y_{2 k}^{*}\right\| \rightarrow 0$. Employing the RMR property for both $f_{i}$ and formula (3.8), we find

$$
\begin{gathered}
\tilde{\varepsilon}_{k} \downarrow 0, \quad\left(x_{1 k}, x_{2 k}\right) \in f^{-1}\left(y_{1 k}, y_{2 k}\right), \\
\left(x_{1 k}^{*}, x_{2 k}^{*}\right) \in \hat{N}_{\tilde{\varepsilon}_{k}}\left(\left(x_{1 k}, x_{2 k}\right) ; f^{-1}(\Omega)\right)
\end{gathered}
$$

such that $\left(x_{1 k}, x_{2 k}\right) \rightarrow\left(\bar{x}_{1}, \bar{x}_{2}\right)$ and $x_{i k}^{*}=\nabla f_{i}\left(\bar{x}_{i}\right)^{*} y_{i k}^{*}$ for $i=1$, 2. By construction, one has $x_{1 k}^{*} \stackrel{w^{*}}{\longrightarrow} 0$ and $\left\|x_{2 k}^{*}\right\| \rightarrow 0$ as $k \rightarrow \infty$. Since $f^{-1}(\Omega)$ is assumed to be PSNC at $\left(\bar{x}_{1}, \bar{x}_{2}\right)$ with respect to $X_{1}$, we conclude that $\left\|x_{1 k}^{*}\right\| \rightarrow 0$ as $k \rightarrow \infty$.

It follows from above that the linear mapping $\nabla f_{1}\left(\bar{x}_{1}\right): X_{1} \rightarrow L$ is metrically regular, where $L=\nabla f_{1}\left(\bar{x}_{1}\right)\left(X_{1}\right)$. This implies that $\left\|\left.y_{1 k}^{*}\right|_{L}\right\| \rightarrow 0$ as $k \rightarrow \infty$. Remember that $L$ is assumed to be of finite codimension; hence it is complemented in $Y_{1}$ by a finitedimensional subspace $L_{1} \subset Y_{1}$. Since $y_{1 k}^{*} \stackrel{w^{*}}{\longrightarrow} 0$, we get that $\left\|\left.y_{1 k}^{*}\right|_{L_{1}}\right\| \rightarrow 0$. Thus $\left\|y_{1 k}^{*}\right\| \rightarrow$ 0 as $k \rightarrow \infty$, which justifies the PSNC property of $\Omega \cap f\left(X_{1} \times X_{2}\right)$ at $\left(\bar{y}_{1}, \bar{y}_{2}\right)$ with respect to $Y_{1}$. The proof of the strong PSNC assertion in (ii) is similar.

REMARK 5.2. Since the SNC property of $\Omega \subset X$ is a special case of the PSNC definition, Theorem 5.1 contains the relationships between the SNC property of sets and their inverse images under strictly differentiable mappings. Furthermore, as it follows from the proof of the above assertion (i) based on the Josefson-Nissenzweig theorem, the SNC property of any $\Omega \subset X$ at some point $\bar{x} \in \Omega$ implies that the closed affine hull of $\Omega$ is finite codimensional in $X$. If $\Omega$ is convex with nonempty relative interior, then one can directly check that the converse implication is also true, that is, the latter two properties are equivalent. Taking into account characterizations of the CEL property for closed convex sets obtained in [2], we now conclude that the SNC and CEL properties agree in Banach spaces for any closed convex sets having closed affine hulls and nonempty relative interiors. Note that the latter assumption is essential even in the framework of Asplund spaces; see [8].

Finally, we present three corollaries of Theorem 5.1 that easily follow from the theorem and the above definitions.

Corollary 5.3. Let $f_{i}: X_{i} \rightarrow Y_{i}, i=1,2$, be mappings between Banach spaces that are strictly differentiable at some points $\bar{x}_{i}$ and such that $\nabla f_{i}(\bar{x})$ are surjective, and let $\Omega$ be a subset of $Y_{1} \times Y_{2}$ with $\left(f_{1}\left(\bar{x}_{1}\right), f_{2}\left(\bar{x}_{2}\right)\right) \in \Omega$. Then $\Omega$ is PSNC (resp., strongly PSNC) at $\left(f_{1}\left(\bar{x}_{1}\right), f_{2}\left(\bar{x}_{2}\right)\right)$ with respect to $Y_{1}$ if and only if $f^{-1}(\Omega)$ is PSNC (resp., strongly PSNC) at $\left(\bar{x}_{1}, \bar{x}_{2}\right)$ with respect to $X_{1}$ for $f=f_{1} \oplus f_{2}$.

Corollary 5.4. Let $f: X \rightarrow Y$ and $G: Y \rightrightarrows Z$ be mappings between Banach spaces, and let $(f(\bar{x}), \bar{z}) \in \operatorname{gph} G$. Assume that $f$ is strictly differentiable at $\bar{x}$ and RMR around this 
point. Then the PSNC (resp., SNC) property of $\left.G\right|_{f}$ at $(f(\bar{x}), \bar{z})$ implies the corresponding property of the composition of $G \circ f$ at $(\bar{x}, \bar{z})$. The opposite implications are also true if, in addition, $\nabla f(\bar{x})(X)$ is assumed to be finite codimensional in $Y$.

COROLLARY 5.5. Let $f: X \rightarrow Y$ be a mapping between Banach spaces that is strictly differentiable at $\bar{x}$ and $R M R$ around this point, and let $\varphi: Y \rightarrow \overline{\mathbb{R}}$ be lower semicontinuous around the point $f(\bar{x})$ where it is finite. Then the SNEC property of $\varphi+\delta_{f(X)}$ at $f(\bar{x})$ implies this property for the composition $\varphi \circ f$ at $\bar{x}$. The opposite implication holds if the space $\nabla f(\bar{x})(X)$ is of finite codimension in $Y$.

ACKNOWLEDGMENTS. The authors are grateful to Alexander Kruger for his valuable remarks and suggestions that allowed us to improve the original presentation. This research was partially supported by the National Science Foundation under Grants DMS9704751 and DMS-0304989.

\section{REFERENCES}

[1] J.-P. Aubin, Lipschitz behavior of solutions to convex minimization problems, Math. Oper. Res. 9 (1984), no. 1, 87-111.

[2] J. M. Borwein, Y. Lucet, and B. S. Mordukhovich, Compactly epi-Lipschitzian convex sets and functions in normed spaces, J. Convex Anal. 7 (2000), no. 2, 375-393.

[3] J. M. Borwein and H. M. Strójwas, Tangential approximations, Nonlinear Anal. 9 (1985), no. $12,1347-1366$.

[4] J. M. Borwein and Q. J. Zhu, A survey of subdifferential calculus with applications, Nonlinear Anal. Ser. A: Theory Methods 38 (1999), no. 6, 687-773.

[5] J. Diestel, Sequences and Series in Banach Spaces, Graduate Texts in Mathematics, vol. 92, Springer-Verlag, New York, 1984.

[6] A. V. Dmitruk, A. A. Milyutin, and N. P. Osmolovskii, Lyusternik's theorem and the theory of extrema, Russian Math. Surveys 35 (1980), no. 6, 11-51.

[7] A. L. Dontchev, A. S. Lewis, and R. T. Rockafellar, The radius of metric regularity, Trans. Amer. Math. Soc. 355 (2003), no. 2, 493-517.

[8] M. Fabian and B. S. Mordukhovich, Sequential normal compactness versus topological normal compactness in variational analysis, Nonlinear Anal. 54 (2003), no. 6, 10571067.

[9] L. M. Graves, Some mapping theorems, Duke Math. J. 17 (1950), 111-114.

[10] A. D. Ioffe, Codirectional compactness, metric regularity and subdifferential calculus, Constructive, Experimental, and Nonlinear Analysis (Limoges, 1999) (M. Théra, ed.), CMS Conf. Proc., vol. 27, Amer. Math. Soc., Rhode Island, 2000, pp. 123-163.

[11] _ Metric regularity and subdifferential calculus, Russian Math. Surveys 55 (2000), no. 3, 501-558.

[12] A. D. Ioffe and V. M. Tihomirov, Theory of Extremal Problems, Studies in Mathematics and Its Applications, vol. 6, North-Holland Publishing, Amsterdam, 1979.

[13] A. Jourani and L. Thibault, Metric regularity and subdifferential calculus in Banach spaces, Set-Valued Anal. 3 (1995), no. 1, 87-100.

[14]__ Coderivatives of multivalued mappings, locally compact cones and metric regularity, Nonlinear Anal. Ser. A: Theory Methods 35 (1999), no. 7, 925-945.

[15] A. Y. Kruger, A covering theorem for set-valued mappings, Optimization 19 (1988), no. 6, 763-780.

[16] P. D. Loewen, Limits of Fréchet normals in nonsmooth analysis, Optimization and Nonlinear Analysis (Haifa, 1990) (A. D. Ioffe, M. Marcus, and S. Reich, eds.), Pitman Res. Notes Math. Ser., vol. 244, Longman Sci. Tech., Harlow, 1992, pp. 178-188.

[17] L. A. Lyusternik, On conditional extrema of functionals, Math. Sbornik 41 (1934), 390-401. 
[18] B. S. Mordukhovich, Approximation Methods in Problems of Optimization and Control, Nauka, Moscow, 1988.

[19] _ Coderivatives of set-valued mappings: calculus and applications, Nonlinear Anal. 30 (1997), no. 5, 3059-3070.

[20] - The extremal principle and its applications to optimization and economics, Optimization and Related Topics (Ballarat/Melbourne, 1999) (A. Rubinov and B. Glover, eds.), Appl. Optim., vol. 47, Kluwer Acad. Publ., Dordrecht, 2001, pp. 343-369.

[21] Calculus of second-order subdifferentials in infinite dimensions, Control Cybernet. 31 (2002), no. 3, 557-573.

[22] B. S. Mordukhovich and J. V. Outrata, On second-order subdifferentials and their applications, SIAM J. Optim. 12 (2001), no. 1, 139-169.

[23] B. S. Mordukhovich and Y. H. Shao, Differential characterizations of covering, metric regularity, and Lipschitzian properties of multifunctions between Banach spaces, Nonlinear Anal. 25 (1995), no. 12, 1401-1424.

[24] _ Nonconvex differential calculus for infinite-dimensional multifunctions, Set-Valued Anal. 4 (1996), no. 3, 205-236.

[25] _ Nonsmooth sequential analysis in Asplund spaces, Trans. Amer. Math. Soc. 348 (1996), no. 4, 1235-1280.

[26] B. S. Mordukhovich and B. Wang, Sequential normal compactness in variational analysis, Nonlinear Anal. 47 (2001), no. 2, 717-728.

[27] __ Extensions of generalized differential calculus in Asplund spaces, J. Math. Anal. Appl. 272 (2002), no. 1, 164-186.

[28] _ Necessary suboptimality and optimality conditions via variational principles, SIAM J. Control Optim. 41 (2002), no. 2, 623-640.

[29] Calculus of sequential normal compactness in variational analysis, J. Math. Anal. Appl. 282 (2003), no. 1, 63-84.

[30] Huynh V. N. and M. Théra, Metric inequality, subdifferential calculus and applications, SetValued Anal. 9 (2001), no. 1-2, 187-216.

[31] J.-P. Penot, Compactness properties, openness criteria and coderivatives, Set-Valued Anal. 6 (1998), no. 4, 363-380.

[32] R. T. Rockafellar, Directionally Lipschitzian functions and subdifferential calculus, Proc. London Math. Soc. (3) 39 (1979), no. 2, 331-355.

[33] R. T. Rockafellar and R. J.-B. Wets, Variational Analysis, Grundlehren der Mathematischen Wissenschaften [Fundamental Principles of Mathematical Sciences], vol. 317, Springer-Verlag, Berlin, 1998.

Boris S. Mordukhovich: Department of Mathematics, Wayne State University, Detroit, MI 48202, USA

E-mail address: boris@math . wayne.edu

Bingwu Wang: Department of Mathematics, Eastern Michigan University, Ypsilanti, MI 48197, USA

E-mail address: bwang@emunix. emich . edu 


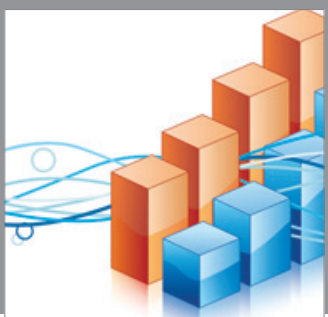

Advances in

Operations Research

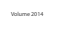

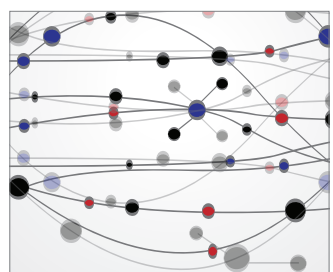

\section{The Scientific} World Journal
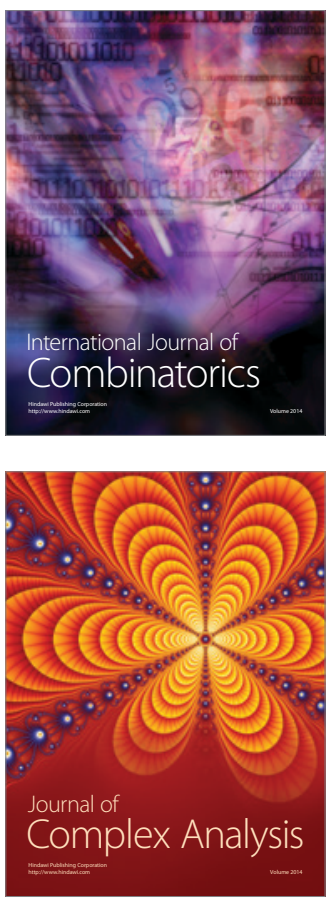

International Journal of

Mathematics and

Mathematical

Sciences
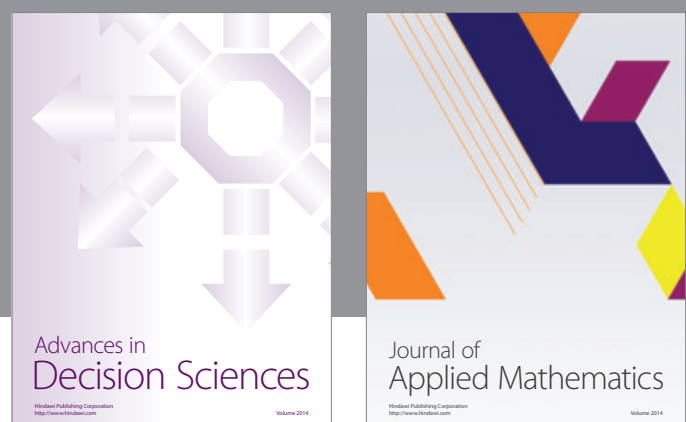

Journal of

Applied Mathematics
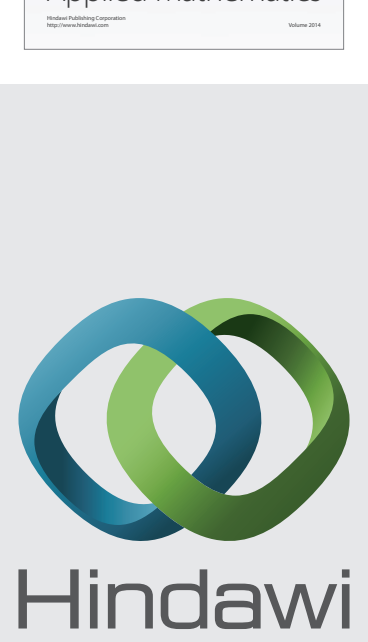

Submit your manuscripts at http://www.hindawi.com
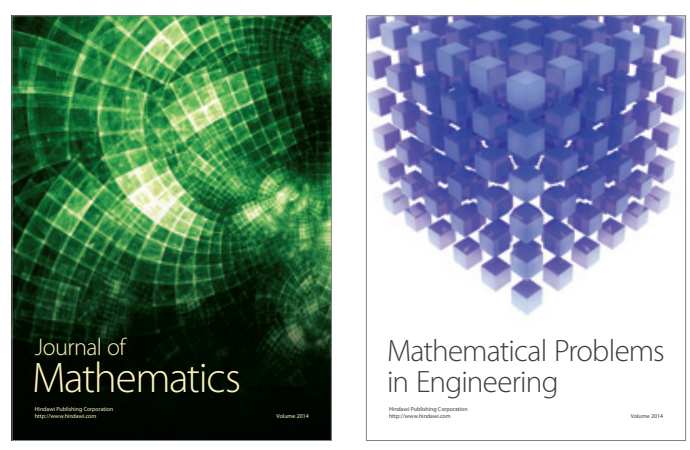

Mathematical Problems in Engineering
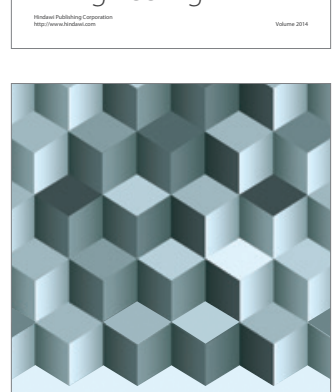

Journal of

Function Spaces
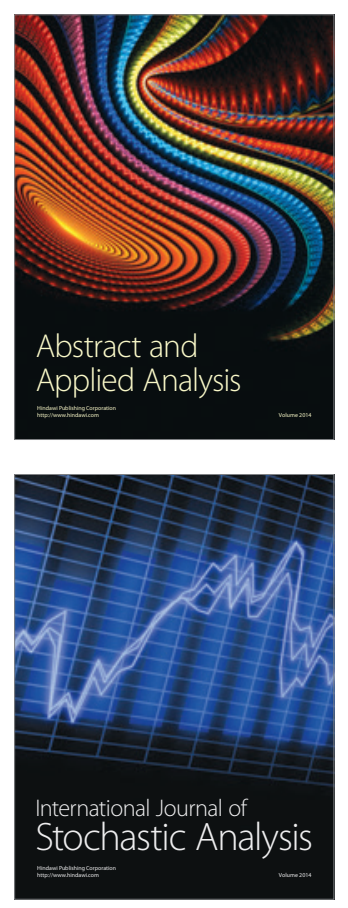

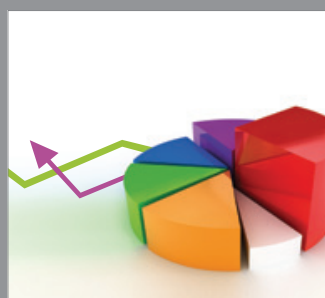

ournal of

Probability and Statistics

Promensencen
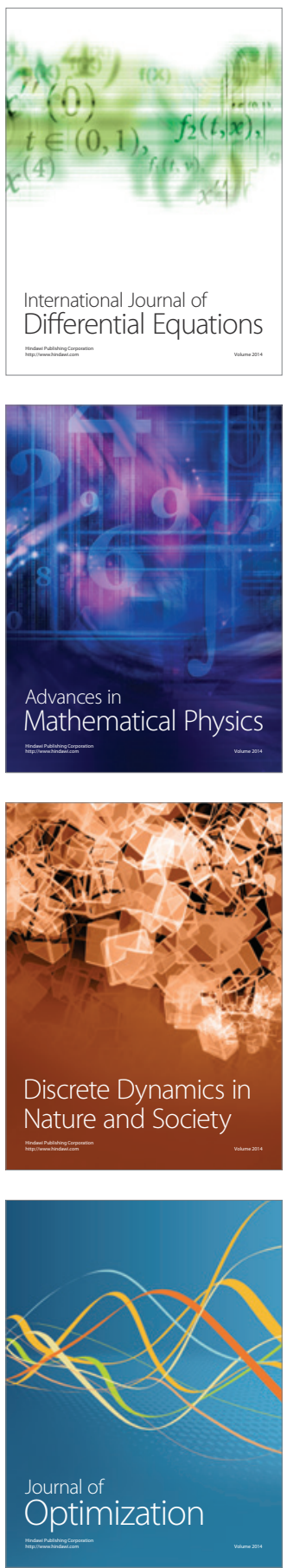